\title{
Supporting the Reconciliation of Models of Object Behaviour ${ }^{1}$
}

\author{
GEORGE SPANOUdAKIS AND HYOSEOB KIM² \\ Department of Computing, \\ City University, \\ Northampton Square, London ECIV OHB, UK \\ E-mail: gespan@soi.city.ac.uk
}

\begin{abstract}
This paper presents Reconciliation+, a method which identifies overlaps between models of software systems behaviour expressed as UML object interaction diagrams (i.e., sequence and/or collaboration diagrams), checks whether the overlapping elements of these models satisfy specific consistency rules and, in cases where they violate these rules, guides software designers in handling the detected inconsistencies. The method detects overlaps between object interaction diagrams by using a probabilistic message matching algorithm that has been developed for this purpose. The guidance to software designers on when to check for inconsistencies and how to deal with them is delivered by enacting a built-in process model that specifies the consistency rules that can be checked against overlapping models and different ways of handling violations of these rules. Reconciliation+ is supported by a toolkit. It has also been evaluated in a case study. This case study has produced positive results which are discussed in the paper.
\end{abstract}

Keywords: consistency management, software design models, object interaction diagrams

\section{Introduction}

The specification of software system behaviour using multiple object interaction diagrams (i.e., sequence and/or collaboration diagrams) creates the potential of conflicting specifications of messages, objects and operations in these models. This is because different object interaction diagrams may, by virtue of the exchanges of messages that they specify and other elements in the specifications of these messages, imply different behaviours for the same objects and operations.

Consider, for example, an object model for a library system that includes the object interaction diagrams $I_{1}$ and $I_{2}$ of Figure 1 and the class diagram of Figure 2. The diagrams $I_{1}$ and $I_{2}$ specify interactions, which occur when the library system is used to search for items in the library either by keywords which refer to the author of an item $\left(\mathrm{I}_{1}\right)$ or by keywords which refer to the title of an item $\left(\mathrm{I}_{2}\right)$. The class diagram of Figure

\footnotetext{
1 This article is an extended version of the article "Reconciliation of Object Interaction Models" that appeared in the proceedings of the $7^{\text {th }}$ International Conference on Object Oriented Information Systems.

2 This article reports on research that was carried out while the second author was affiliated with the Department of Computing of City University.
} 
2 specifies the classes of the objects that participate in the interactions of $I_{1}$ and $I_{2}$. According to $\mathrm{I}_{1}$ and $\mathrm{I}_{2}$, the library system: (i) gets search keywords from a UI component (see messages 11:getText () in $\mathrm{I}_{1}$ and 8 :getText () in $\mathrm{I}_{2}$ ); (ii) formulates a database query (see message 9 : formulateQuery () in $\mathrm{I}_{2}$ ); and (iii) executes the query (see messages 12 : executeQuery (SQLStatement) in $I_{1}$ and 10 : executeQuery (SQLSt) in $\mathrm{I}_{2}$ ).

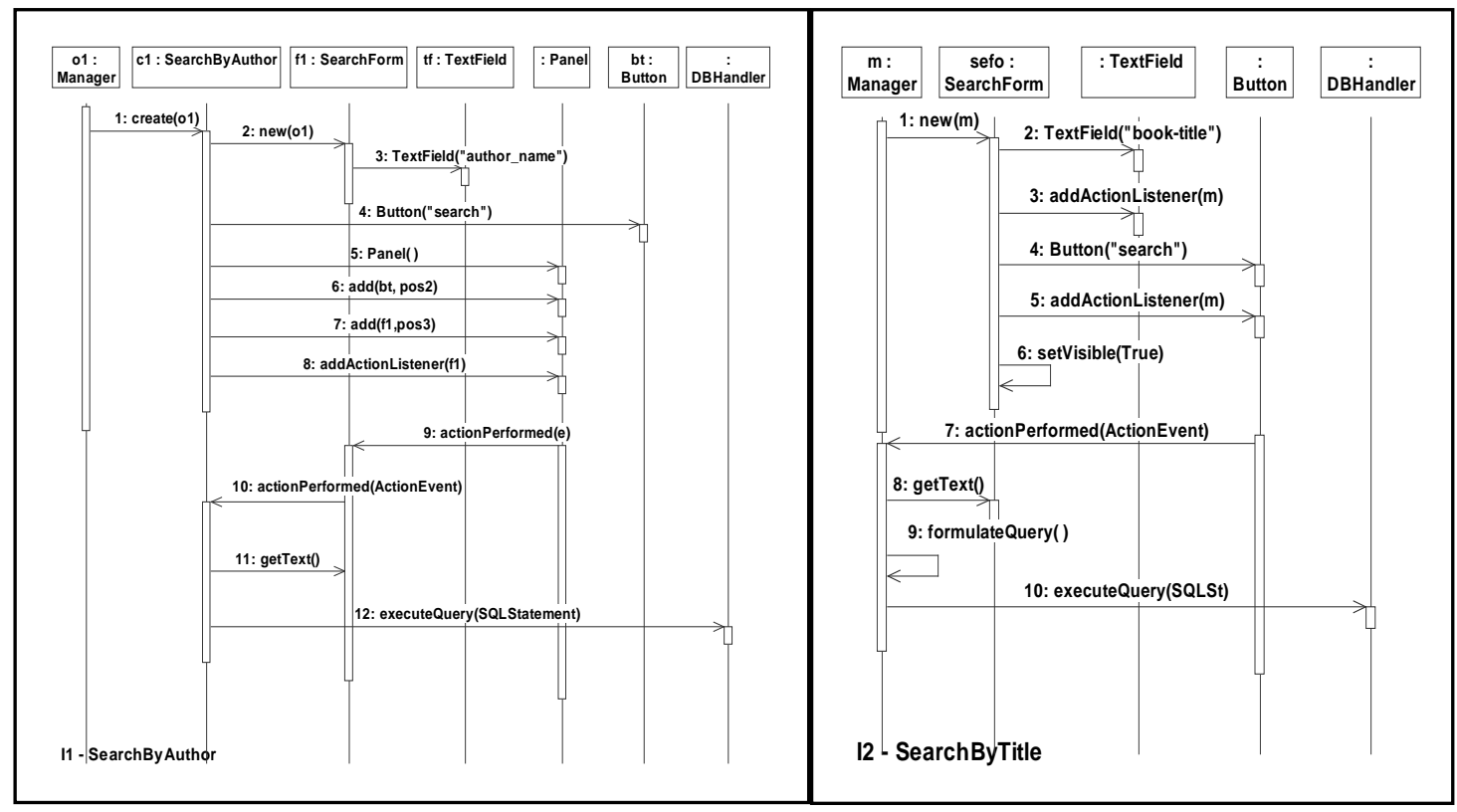

Figure 1: Object interaction diagrams SearchByAuthor $\left(\mathrm{I}_{1}\right)$ and SearchByTit le $\left(\mathrm{I}_{2}\right)$

In this example, it is plausible to assume that the messages 10: actionPerformed (ActionEvent) in $\mathrm{I}_{1}$ and 7 :actionPerformed (ActionEvent) in $\mathrm{I}_{2}$ overlap since (in the current state of the models) they both appear to invoke the operation actionPerformed (e:ActionEvent) of the class DatabaseActionListener in Figure 2. If, however, this assumption is correct then the specifications of the behaviour of the operation actionPerformed(e:ActionEvent) which are implied by $I_{1}$ and $I_{2}$ are conflicting. This is because according to $\mathrm{I}_{2}$ (but not $\mathrm{I}_{1}$ ) the execution of the operation actionPerformed(e:ActionEvent) leads to the dispatch of the message 9:formulatequery() and therefore the execution of the operation formulateQuery () of the class Manager.

Conflicts of this form need to be detected and reconciled in the design phase of a system development project in order to eliminate ambiguities that could be more expensive to resolve at the implementation phase.

In this paper, we describe a tool-supported method, called Reconciliation+, that we have developed to support software designers to detect and handle conflicts in behavioural specifications in models of object interactions which are expressed as sequence (or collaboration) diagrams in UML [9]. This method is a newly developed 
extension of a method for managing modelling discrepancies in structural object models that is discussed in [15].

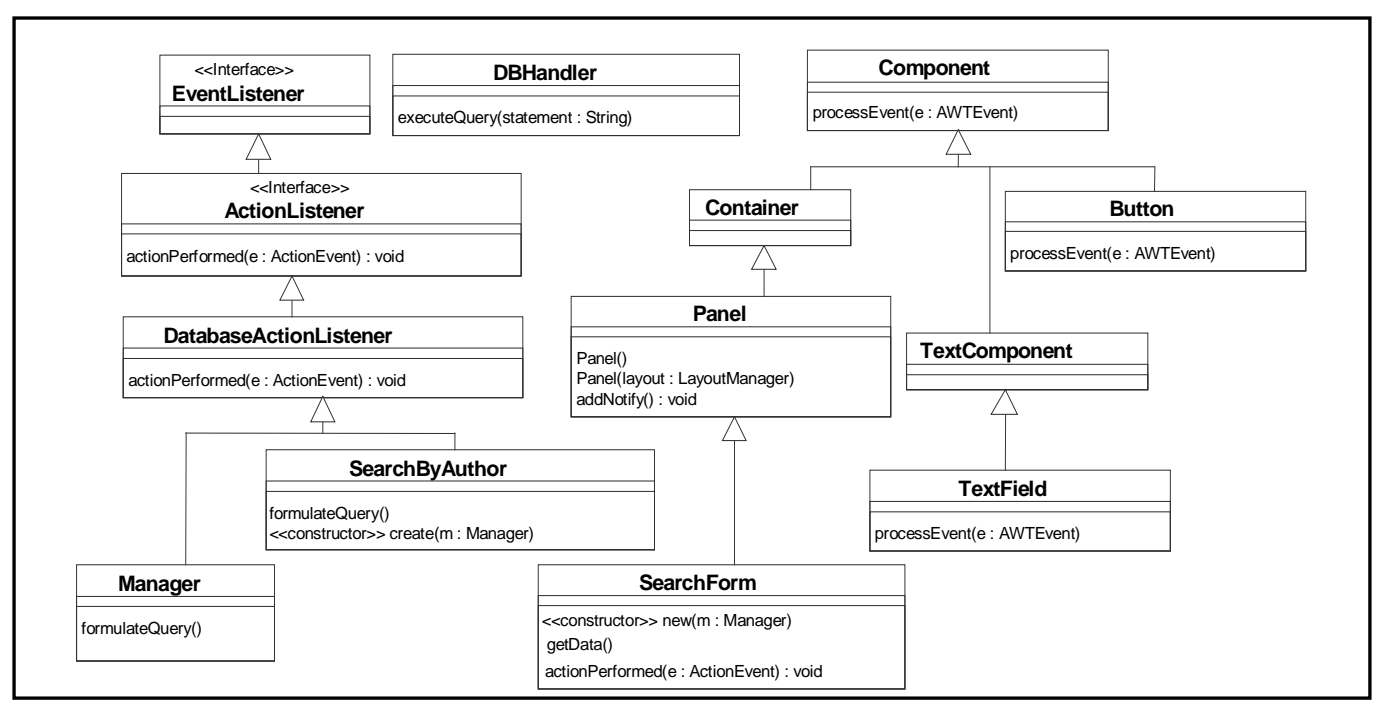

Figure 2: Model of classes of object interaction diagrams of Figure 1

Conflicts in Reconciliation+ are detected as violations of consistency rules. A consistency rule sets the conditions that should be satisfied by overlapping messages in different object interaction diagrams. In the above example of the library system, for instance, the conflict in the specifications of the behaviour of the operation actionPerformed (e:ActionEvent) implied by $\mathrm{I}_{1}$ and $\mathrm{I}_{2}$ could be detected as a violation of the following consistency rule:

CR1: If a message $m_{i}$ overlaps with a message $m_{j}$ then for every message $m_{k}$ that is dispatched by $m_{i}\left(m_{j}\right)$ there must exist a message $m_{w}$ dispatched by $m_{j}\left(m_{i}\right)$ such that $m_{k}$ and $m_{w}$ overlap.

CR1 in this case would be violated by the message 9 :formulateQuery () that is dispatched by the message 7 :actionPerformed (ActionEvent) in $I_{2}$ and has no overlapping counterpart in the messages which are dispatched by the message 10 : actionPerformed (ActionEvent) in $I_{1}$ that overlaps with it.

Overlaps in Reconciliation+ are defined as relations between messages which are likely to signify the invocation of operations with the same implementation and are detected by a probabilistic message matching algorithm. This algorithm can detect overlaps between messages which are not identically modelled if certain parts of their specifications provide strong evidence to believe that the messages signify the invocation of operations with the same implementation. Thus, for example, it may detect an overlap relation between two messages $m_{i}$ and $m_{j}$ that have different signatures if they are sent to objects which are instances of the same class and dispatch the same messages (e.g. message 12 : executeQuery (SQLStatement) in $I_{1}$ and message 10 : executeQuery (SQLSt) in $\mathrm{I}_{2}$ ). 
Reconciliation+ incorporates a set of pre-defined consistency rules that should be satisfied by overlapping messages and alternative ways of handling violations of these rules. The method guides software designers in selecting which of these consistency rules to check and how to handle their violations. This guidance is delivered by enacting a built-in process model that specifies the consistency rules that can be checked against overlapping messages, and different ways of handling the violations of these rules. The method is extensible as software designers can extend its built-in process model by specifying new consistency rules, and new ways of handling violations of new or existing rules.

The rest of this paper is structured as follows. In Section 2, we describe the algorithm that Reconciliation+ deploys for detecting overlapping messages in different interaction diagrams. In Section 3, we describe the specification and enactment of the process model of the method that is used for guiding designers in reconciling object interaction diagrams. In Section 4, we describe the specification of consistency rules using the process model of the method and the mechanism for detecting violations of these rules. In Section 5, we describe the scheme for specifying and executing different ways of handling inconsistencies. In Section 6, we present an overview of the prototype toolkit we have developed to support Reconciliation+. In Section 7, we present the results of a case study that we have conducted to evaluate the method. Finally, in Section 8 we overview related work and, in Section 9, we summarise the method and outline ongoing work on it.

\section{Detection of overlaps}

\subsection{Basic algorithmic formulation}

Overlaps in Reconciliation+ are defined as relations between messages which are likely to signify the invocation of operations with the same implementation. The detection of these relations is formulated as an instance of the weighted bipartite graph matching problem [10]. More specifically, assuming a pair of interaction diagrams $\mathrm{I}_{\mathrm{i}}$ and $\mathrm{I}_{\mathrm{j}}$, we construct a weighted interaction overlap graph: $\operatorname{IOG}\left(\mathrm{I}_{\mathrm{i}}, \mathrm{I}_{\mathrm{j}}\right)=\left(\mathrm{V}_{\mathrm{i}}\right.$ $\cup V_{j}, E\left(V_{i}, V_{j}\right)$ ). This graph has two sets of disjoint vertices, $V_{i}$ and $V_{j}$, which assuming that $\mathrm{I}_{\mathrm{i}}$ has more messages than $\mathrm{I}_{\mathrm{j}}$, are defined as:

$$
\mathrm{V}_{\mathrm{i}} \equiv \operatorname{Messages}\left(\mathrm{I}_{\mathrm{i}}\right) \text { and } \mathrm{V}_{\mathrm{j}} \equiv \operatorname{Messages}\left(\mathrm{I}_{\mathrm{j}}\right) \cup \mathrm{DV}_{\mathrm{k}}
$$

where

- Messages $\left(I_{i}\right)$ is the set of messages of the interaction diagram $\mathrm{I}_{\mathrm{i}}$;

- Messages $\left(I_{j}\right)$ is the set of messages of the interaction diagram $I_{j}$; and

- $\mathrm{DV}_{\mathrm{k}}$ is a set of $\mathrm{k}$ special vertices representing dummy messages $\left(\mathrm{k}=\left|\operatorname{Messages}\left(\mathrm{I}_{\mathrm{i}}\right)\right|\right.$ $\left.-\left|\operatorname{Messages}\left(\mathrm{I}_{\mathrm{j}}\right)\right|\right)$.

The set of the edges $\mathrm{E}\left(\mathrm{V}_{\mathrm{i}}, \mathrm{V}_{\mathrm{j}}\right)$ includes all the possible edges between the messages of $I_{i}$ and the messages of $I_{j}$, or formally:

$$
\mathrm{E}\left(\mathrm{V}_{\mathrm{i}}, \mathrm{V}_{\mathrm{j}}\right)=\left\{\left(\mathrm{n}_{\mathrm{i}}, \mathrm{n}_{\mathrm{j}}, \mathrm{b}_{0}\left(\neg \mathrm{OV}\left(\mathrm{n}_{\mathrm{i}}, \mathrm{n}_{\mathrm{j}}\right)\right)\right) \mid\left(\mathrm{n}_{\mathrm{i}} \in \mathrm{V}_{\mathrm{i}}\right) \text { and }\left(\mathrm{n}_{\mathrm{j}} \in \mathrm{V}_{\mathrm{j}}\right)\right\}
$$


An edge $\left(\mathrm{n}_{\mathrm{i}}, \mathrm{n}_{\mathrm{j}}, \mathrm{b}_{0}\left(\neg \mathrm{ov}\left(\mathrm{n}_{\mathrm{i}}, \mathrm{n}_{\mathrm{j}}\right)\right)\right)$ in $\mathrm{E}\left(\mathrm{V}_{\mathrm{i}}, \mathrm{V}_{\mathrm{j}}\right)$ designates the assumption that the messages represented by the nodes $n_{i}$ and $n_{j}$ overlap and is weighted by the measure $\mathrm{b}_{0}\left(\neg \mathrm{ov}\left(\mathrm{n}_{\mathrm{i}}, \mathrm{n}_{\mathrm{j}}\right)\right)$. This measure is defined as the degree of belief in the falsity of the overlap assumption expressed by the edge, and is computed according to the following function:

$$
\begin{aligned}
& \mathrm{b}_{0}\left(\neg \mathrm{OV}\left(\mathrm{n}_{\mathrm{i}}, \mathrm{n}_{\mathrm{j}}\right)\right)=\Sigma_{\mathrm{U} \subseteq\{1, \ldots, 6\}}(-1)^{|\mathrm{U}|+1}\left\{\Pi_{\mathrm{u} \in \mathrm{U}} \mathrm{b}_{\mathrm{u}}\left(\neg \mathrm{OV}\left(\mathrm{n}_{\mathrm{i}}, \mathrm{n}_{\mathrm{j}}\right)\right)\right\} \\
& \text { if } \mathrm{n}_{\mathrm{i}} \in \operatorname{Messages}\left(\mathrm{I}_{\mathrm{i}}\right) \text { and } \mathrm{n}_{\mathrm{j}} \in \operatorname{Messages}\left(\mathrm{I}_{\mathrm{j}}\right) \\
& \mathrm{b}_{0}\left(\neg \mathrm{ov}\left(\mathrm{n}_{\mathrm{i}}, \mathrm{n}_{\mathrm{j}}\right)\right)=1 \text { if } \mathrm{n}_{\mathrm{j}} \in \mathrm{DV}_{\mathrm{k}}
\end{aligned}
$$

The functions $b_{1}, \ldots, b_{6}$ used in (I) compute partial beliefs in the existence/absence of an overlap between two messages. The computation of these partial beliefs is based on heuristic criteria for assessing the equivalence of the functional roles and implementations of the operations invoked by the messages, and the functional contexts in which these operations are invoked. These belief functions and the criteria underpinning them are discussed in detail in Section 2.2.

After computing the beliefs $b_{0}$ for all the edges of $\operatorname{IOG}\left(\mathrm{I}_{\mathrm{i}}, \mathrm{I}_{\mathrm{j}}\right)$, the most likely overlaps between the messages in $I_{i}$ and $I_{j}$ are detected in two steps. In the first step, the most likely candidate overlaps are identified by selecting a subset $\mathrm{O}\left(\mathrm{V}_{\mathrm{i}}, \mathrm{V}_{\mathrm{j}}\right)$ of $\mathrm{E}\left(\mathrm{V}_{\mathrm{i}}, \mathrm{V}_{\mathrm{j}}\right)$ which is a total morphism between $V_{i}$ and $V_{j}$ and minimises the function ${ }^{3}$ :

$$
\Sigma_{(\mathrm{nu}, \mathrm{nw}, \mathrm{b} 0(\neg \mathrm{ov}(\mathrm{nu}, \mathrm{nw}))) \in \mathrm{O}(\mathrm{Vi}, \mathrm{Vj})} \mathrm{b}_{0}\left(\neg \mathrm{OV}\left(\mathrm{n}_{\mathrm{u}}, \mathrm{n}_{\mathrm{w}}\right)\right)
$$

In the second step, $\mathrm{O}\left(\mathrm{V}_{\mathrm{i}}, \mathrm{V}_{\mathrm{j}}\right)$ is restricted to include only the edges $\left(\mathrm{n}_{\mathrm{u}}, \mathrm{n}_{\mathrm{w}}, \mathrm{b}_{0}\left(\neg \mathrm{Ov}\left(\mathrm{n}_{\mathrm{u}}, \mathrm{n}_{\mathrm{w}}\right)\right)\right.$ whose belief does not exceed a threshold value $\mathrm{b}_{\mathrm{t}}$, (i.e., edges for which $\left.b_{0}\left(\neg \mathrm{ov}\left(\mathrm{n}_{\mathrm{u}}, \mathrm{n}_{\mathrm{v}}\right)\right) \leq \mathrm{b}_{\mathrm{t}}\right)$.

\subsection{Criteria of overlap and partial belief functions}

Beliefs in favour of, or against a hypothesis that two messages overlap are computed based on six criteria. These criteria indicate the equivalence of the functional roles and implementations of the operations which are invoked by the messages, and the equivalence of the contexts in which these operations are invoked.

\subsubsection{Equivalence of functional roles of invoked operations}

Beliefs in the equivalence of the functional roles of the operations which are invoked by two messages are computed using two criteria, namely the criterion of the most generic overridden operation and the criterion of the operation stereotypes.

\section{The criterion of most generic overridden operation}

Two operations are assumed to have equivalent functional roles if they override the same most general operation in an object model.

According to this criterion, the operations processEvent (e: AWTEvent) which are defined in the classes Button and TextField in Figure 2, for example, are

\footnotetext{
${ }^{3}$ The morphism $\mathrm{O}\left(\mathrm{V}_{\mathrm{i}}, \mathrm{V}_{\mathrm{j}}\right)$ is selected using the Hungarian method [10].
} 
considered to have equivalent functional roles. This is because both of them override the same most generic operation in the class model of the figure, namely the operation processEvent (e:AWTEvent) of Component. In this example, the classes Button and TextField override the latter operation that they inherit from Component in order to introduce the different functionality which is required for processing events of two different types: action events by Button and text events by TextField [20]. Despite of the differences in their exact functionality, however, the functional role of the operations processEvent (AWTEvent e) in Button and TextField is the same, that is to enable the instances of these classes to handle events related to them.

Note that, while in single inheritance hierarchies an operation always overrides a single most generic operation (which may trivially be itself ${ }^{4}$ ), in multiple inheritance graphs there is a potential for ambiguity. Such an ambiguity arises in cases where two superclasses of a class $\mathrm{C}$, which are not directly or implicitly related by a generalisation relation themselves, define operations that have the same signature as an operation that is defined in $\mathrm{C}$.

To cope with ambiguities of this form, we introduce the following function that measures the degree of belief in the existence (absence) of an overlap relation between two messages based on the criterion of the most generic overridden operations:

Definition 1: The degree of belief in the existence (absence) of an overlap relation between two messages $m_{i}$ and $m_{j}$ based on the criterion of the most generic operations overridden by the operations invoked by $\mathrm{m}_{\mathrm{i}}$ and $\mathrm{m}_{\mathrm{j}}$ is computed according to the function:

where

$$
\mathrm{b}_{1}\left(\neg \mathrm{ov}\left(\mathrm{m}_{\mathrm{i}}, \mathrm{m}_{\mathrm{j}}\right)\right)=\alpha_{1} \times \mathrm{d}_{1}\left(\mathrm{~m}_{\mathrm{i}}, \mathrm{m}_{\mathrm{j}}\right) \quad \text { and } \quad \mathrm{b}_{1}\left(\mathrm{ov}\left(\mathrm{m}_{\mathrm{i}}, \mathrm{m}_{\mathrm{j}}\right)\right)=0
$$

- $\mathrm{d}_{1}\left(\mathrm{~m}_{\mathrm{i}}, \mathrm{m}_{\mathrm{j}}\right)=\left(\left|\mathrm{Os}\left(\mathrm{o}_{\mathrm{i}}\right)-\mathrm{Os}\left(\mathrm{o}_{\mathrm{j}}\right)\right|+\left|\mathrm{Os}\left(\mathrm{o}_{\mathrm{j}}\right)-\mathrm{Os}\left(\mathrm{o}_{\mathrm{i}}\right)\right|\right) /\left|\operatorname{Os}\left(\mathrm{o}_{\mathrm{i}}\right) \cup \mathrm{Os}\left(\mathrm{o}_{\mathrm{j}}\right)\right|$

if $\operatorname{Os}\left(\mathrm{o}_{\mathrm{i}}\right) \neq \varnothing$ and $\mathrm{Os}\left(\mathrm{o}_{\mathrm{j}}\right) \neq \varnothing$

- $\mathrm{d}_{1}\left(\mathrm{~m}_{\mathrm{i}}, \mathrm{m}_{\mathrm{j}}\right)=1$

if $\operatorname{Os}\left(\mathrm{o}_{\mathrm{i}}\right)=\varnothing$ or $\operatorname{Os}\left(\mathrm{o}_{\mathrm{j}}\right)=\varnothing$

- $\mathrm{o}_{\mathrm{i}}$ is the operation invoked by $\mathrm{m}_{\mathrm{i}}$ and $\mathrm{o}_{\mathrm{j}}$ is the operation invoked by $\mathrm{m}_{\mathrm{j}}$

- Os $\left(\mathrm{o}_{\mathrm{i}}\right)\left(\mathrm{Os}\left(\mathrm{o}_{\mathrm{j}}\right)\right)$ is a set of operations which are defined in the superclasses of the class that defines $o_{i}\left(o_{j}\right)$, have the same signature with it, and do not override any other operation with the same signature.

- $\alpha_{1}$ is the expected ratio of messages that invoke operations which do not override the same most generic operation and do not overlap $\left(0 \leq \alpha_{1} \leq 1\right)$

\section{Examples}

The belief produced by $b_{1}$ in the absence of an overlap relation between the messages 7 :actionPerformed (ActionEvent) and 10 :actionPerformed (ActionEvent)) in the interaction diagrams $I_{1}$ and $I_{2}$ of Figure 1 is 0 . This is because, according to the class model of Figure 2, both these messages invoke the same operation, that is DatabaseActionListener.actionPerformed(e:ActionEvent), and

\footnotetext{
${ }^{4}$ As in the case of the operation formulateQuery () of the class Manager in Figure 2.
} 
therefore the same most general overridden operation (i.e., ActionListener.actionPerformed (e:ActionEvent)). Thus, the $d_{1}$ distance between these messages is 0 .

Note, however, that the belief in the absence of an overlap relation between the messages 9:actionPerformed (e) in $\mathrm{I}_{1}$ and 7 :actionPerformed (ActionEvent) in $I_{2}$ that is generated by $b_{1}$ is 0.4 (assuming that $\alpha_{1}=0.4$ ). This is because the former message invokes the operation SearchForm.actionPerformed(e: ActionEvent) and the latter message invokes the operation DatabaseActionListener.actionPerformed (e: ActionEvent) in Figure 2. And as these two operations override different most general operations in the relevant class model (i.e., the operation SearchForm.actionPerformed(e: ActionEvent) and the operation Actiontistener.actionPerformed (e:ActionEvent), respectively), the distance between the above messages is 1 . As a result, $b_{1}$ assumes that the functional roles of SearchForm.actionPerformed(e: ActionEvent) and DatabaseActionListener.actionPerformed(e: ActionEvent) are different and, therefore, it generates the maximum possible belief against the existence of an overlap relation between 9:actionPerformed(e) and 7 : actionPerformed (ActionEvent) .

\section{The criterion of operation stereotypes}

The second criterion for assessing the equivalence of the functional roles of two operations is based on operation stereotypes. According to this criterion, the functional roles of two operations are considered equivalent if the operations have the same stereotype(s). This criterion is used since, in UML, operation stereotypes are used to designate groups of functionally similar operations. The belief function associated with this criterion is defined as follows:

Definition 2: The degree of belief in the existence (absence) of an overlap relation between two messages $m_{i}$ and $m_{j}$ based on the criterion of the stereotypes of the operations that $\mathrm{m}_{\mathrm{i}}$ and $\mathrm{m}_{\mathrm{j}}$ invoke is computed according to the function:

where

$$
\mathrm{b}_{4}\left(\neg \mathrm{ov}\left(\mathrm{m}_{\mathrm{i}}, \mathrm{m}_{\mathrm{j}}\right)\right)=\alpha_{4} \times \mathrm{d}_{4}\left(\mathrm{~m}_{\mathrm{i}}, \mathrm{m}_{\mathrm{j}}\right) \quad \text { and } \quad \mathrm{b}_{4}\left(\mathrm{ov}\left(\mathrm{m}_{\mathrm{i}}, \mathrm{m}_{\mathrm{j}}\right)\right)=0
$$

- $\mathrm{d}_{4}\left(\mathrm{~m}_{\mathrm{i}}, \mathrm{m}_{\mathrm{j}}\right)=\left(\left|\operatorname{St}\left(\mathrm{o}_{\mathrm{i}}\right)-\operatorname{St}\left(\mathrm{o}_{\mathrm{j}}\right)\right|+\left|\operatorname{St}\left(\mathrm{o}_{\mathrm{j}}\right)-\operatorname{St}\left(\mathrm{o}_{\mathrm{i}}\right)\right|\right) /\left|\operatorname{St}\left(\mathrm{o}_{\mathrm{i}}\right) \cup \operatorname{St}\left(\mathrm{o}_{\mathrm{j}}\right)\right| \quad$ if $\operatorname{St}\left(\mathrm{o}_{\mathrm{i}}\right) \neq \varnothing$ and $\operatorname{St}\left(\mathrm{o}_{\mathrm{j}}\right) \neq \varnothing$

- $\mathrm{d}_{4}\left(\mathrm{~m}_{\mathrm{i}}, \mathrm{m}_{\mathrm{j}}\right)=1 \quad$ if $\operatorname{St}\left(\mathrm{o}_{\mathrm{i}}\right)=\varnothing$ or $\operatorname{St}\left(\mathrm{o}_{\mathrm{j}}\right)=\varnothing$

- $\mathrm{o}_{\mathrm{i}}$ is the operation invoked by $\mathrm{m}_{\mathrm{i}}$ and $\mathrm{o}_{\mathrm{j}}$ is the operation invoked by $\mathrm{m}_{\mathrm{j}}$

- $\operatorname{St}\left(\mathrm{o}_{\mathrm{i}}\right)$ and $\operatorname{St}\left(\mathrm{o}_{\mathrm{j}}\right)$ are the sets of the stereotypes of $\mathrm{o}_{\mathrm{i}}$ and $\mathrm{o}_{\mathrm{j}}$

- $\alpha_{4}$ is the expected ratio of operations with different stereotypes which do not overlap $\left(0 \leq \alpha_{4} \leq 1\right)$

The functional form of $b_{4}$ covers cases where an operation may belong to different stereotype groups. In these cases, $\mathrm{d}_{4}$ measures the likelihood of the operations invoked by two messages not having a common stereotype. 


\section{Examples}

The $\mathrm{d}_{4}$ distance between the messages 1 : create $(\mathrm{ol})$ in $\mathrm{I}_{1}$ and 1 : new $(\mathrm{m})$ in $\mathrm{I}_{2}$ is 0 . This is because the operations SearchByAuthor.create (o:Manager) and SearchForm. new (o: SearchByAuthor) which are invoked by these messages, are both stereotyped as constructor-operations in the class diagram of Figure 2. Thus, $\mathrm{b}_{4}$ is also 0 in this case. For any other pair of messages in Figure 1, however, since the stereotypes of the operations invoked by them are not defined (see Figure 2) the $\mathrm{d}_{4}$ distance is 1 and thus it generates a $b_{4}$ belief equal to $\alpha_{4}$.

\subsubsection{Equivalence of the functional contexts of operations}

The assessment of the equivalence of the functional contexts of two messages is based on three criteria. The first of these criteria is whether the messages are sent by instances of the same class or, equivalently in terms of UML, they have the same senders. The second criterion is whether the messages are received by instances of the same class or, in terms of UML, they have the same receivers. The third criterion is whether the messages are dispatched by the same message or, in terms of UML, have the same activator.

\section{The criterion of message senders}

According to this criterion, the functional contexts in which two messages are dispatched are considered to be different if the messages are sent by objects which are instances of different classes. The belief function that is associated with this criterion is defined as:

Definition 3: The degree of belief in the existence (absence) of an overlap relation between two messages $\mathrm{m}_{\mathrm{i}}$ and $\mathrm{m}_{\mathrm{j}}$ based on the criterion of message senders is computed according to the function:

where

$$
\mathrm{b}_{2}\left(\neg \mathrm{Ov}\left(\mathrm{m}_{\mathrm{i}}, \mathrm{m}_{\mathrm{j}}\right)\right)=\alpha_{2} \times \mathrm{d}_{2}\left(\mathrm{~s}_{\mathrm{i}}, \mathrm{s}_{\mathrm{j}}\right) \quad \text { and } \quad \mathrm{b}_{2}\left(\mathrm{ov}\left(\mathrm{m}_{\mathrm{i}}, \mathrm{m}_{\mathrm{j}}\right)\right)=0
$$

- $\mathrm{s}_{\mathrm{i}}$ and $\mathrm{s}_{\mathrm{j}}$ are the classes of the objects that send $\mathrm{m}_{\mathrm{i}}$ and $\mathrm{m}_{\mathrm{j}}$, respectively

- $\mathrm{d}_{2}\left(\mathrm{~s}_{\mathrm{i}}, \mathrm{s}_{\mathrm{j}}\right)$ is a function measuring the generalisation distance between $\mathrm{s}_{\mathrm{i}}$ and $\mathrm{s}_{\mathrm{j}}$ defined as:

$$
\begin{array}{ll}
-\mathrm{d}_{2}\left(\mathrm{~s}_{\mathrm{i}}, \mathrm{s}_{\mathrm{j}}\right)=\sum_{\mathrm{x} \in \mathrm{NCSij}} \mathrm{SD}(\mathrm{x})^{-1} / \sum_{\mathrm{y} \in \mathrm{ASSij}} \mathrm{SD}(\mathrm{y})^{-1} & \text { if } \mathrm{s}_{\mathrm{i}} \text { and } \mathrm{s}_{\mathrm{i}} \text { are specified } \\
\mathrm{d}_{2}\left(\mathrm{~s}_{\mathrm{i}}, \mathrm{s}_{\mathrm{j}}\right)=1 & \text { if } \mathrm{s}_{\mathrm{i}} \text { or } \mathrm{s}_{\mathrm{i}} \text { is not specified }
\end{array}
$$

$-\operatorname{NCS}_{\mathrm{ij}}=\left(\left(\mathrm{s}_{\mathrm{i}}\right.\right.$.Isa* $\left.\cup\left\{\mathrm{s}_{\mathrm{i}}\right\}\right)-\left(\mathrm{s}_{\mathrm{j}}\right.$.Isa* $\left.\left.\cup\left\{\mathrm{s}_{\mathrm{j}}\right\}\right)\right) \cup\left(\left(\mathrm{s}_{\mathrm{j}}\right.\right.$.Isa* $\left.\cup\left\{\mathrm{s}_{\mathrm{j}}\right\}\right)-\left(\mathrm{s}_{\mathrm{i}}\right.$.Isa* $\left.\left.\cup\left\{\mathrm{s}_{\mathrm{i}}\right\}\right)\right)$

$-\mathrm{ASS}_{\mathrm{ij}}=\left(\left(\mathrm{s}_{\mathrm{i}}\right.\right.$.Isa* $\left.\cup\left\{\mathrm{s}_{\mathrm{i}}\right\}\right) \cup\left(\mathrm{s}_{\mathrm{j}}\right.$.Isa* $\left.\left.\cup\left\{\mathrm{s}_{\mathrm{j}}\right\}\right)\right)$

- $\mathrm{SD}(\mathrm{x})$ is the length of the longest path connecting a class $\mathrm{x}$ with its most general superclass, called specialisation depth of $\mathrm{x}$

- $\mathrm{s}_{\mathrm{i}}$.Isa* $\left(\mathrm{s}_{\mathrm{j}}\right.$.Isa*) is the transitive closure of the superclasses of class $\mathrm{s}_{\mathrm{i}}\left(\mathrm{s}_{\mathrm{j}}\right)$

- $\alpha_{2}$ is the expected ratio of non overlapping messages with different senders $\left(0 \leq \alpha_{2} \leq\right.$ 1)

The assumption underpinning the definition of the belief function $b_{2}$ is that the identity of class names is not necessarily an accurate indicator of the identity of classes, especially if classes are specified in two independently constructed models of the same 
system. Thus, $\mathrm{b}_{2}$ computes a belief in class identity based not only on the classes themselves but also on their superclasses. According to its definition, the more the non-common superclasses of two classes $c_{i}$ and $c_{j}$ the stronger the belief that $c_{i}$ and $c_{j}$ are not identical.

Each of the non-common superclasses of two classes $c_{i}$ and $c_{j}$ produces evidence of different strength for the assumption that $c_{i}$ and $c_{j}$ are not identical. The strength of this evidence is measured as the inverse of the specialisation depth (i.e., the length of the longest path connecting a class with its most general superclasses) of a superclass in the generalisation graph of the model(s). According to this measure, non common superclasses which appear in relatively low levels of generalisation graphs and, by virtue of their position, introduce fine-grain specialisations of more general classes provide weaker evidence than classes which appear in higher positions [14].

\section{Examples}

Given the class model of Figure 2, the $\mathrm{d}_{2}$ distance between the classes Manager and SearchByAuthor that send the messages 2 : new $(o 1)$ and 1 :new $(\mathrm{m})$ in the interaction diagrams $I_{1}$ and $I_{2}$ is 0.21 . Thus, assuming that $\alpha_{2}=0.1$, the belief in the absence of an overlap relation between these messages that $b_{2}$ generates is 0.021 .

\section{The criterion of message receivers}

According to this criterion, the functional contexts of two messages are not considered to be equivalent if the messages are received by objects which are instances of different classes or, equivalently in terms of UML, if they have different receivers. The belief function that is associated with this criterion is defined as:

Definition 4: The degree of belief in the existence (absence) of an overlap relation between two messages $\mathrm{m}_{\mathrm{i}}$ and $\mathrm{m}_{\mathrm{j}}$ based on the criterion of message receivers is computed according to the function:

where

$$
\mathrm{b}_{3}\left(\neg \mathrm{ov}\left(\mathrm{m}_{\mathrm{i}}, \mathrm{m}_{\mathrm{j}}\right)\right)=\alpha_{3} \times \mathrm{d}_{2}\left(\mathrm{r}_{\mathrm{i}}, \mathrm{r}_{\mathrm{j}}\right) \quad \text { and } \quad \mathrm{b}_{3}\left(\mathrm{ov}\left(\mathrm{m}_{\mathrm{i}}, \mathrm{m}_{\mathrm{j}}\right)\right)=0
$$

- $r_{i}$ and $r_{j}$ are the classes of the objects that receive $m_{i}$ and $m_{j}$, respectively

- $\mathrm{d}_{2}$ is as defined in Definition 3

- $\alpha_{3}$ is the expected ratio of messages with different receivers which do not overlap $\left(0 \leq \alpha_{3} \leq 1\right)$

The rational for using the above function for measuring belief in the absence/existence of an overlap relation between the receivers of two messages is the same as that used in the case of the function $b_{2}$.

\section{The criterion of message activators}

The third criterion for calculating belief in the equivalence of the functional contexts of two messages $m_{i}$ and $m_{j}$ is whether these messages are sent by messages which overlap themselves (these messages are called activators in UML). The belief function associated with this criterion of message activators is defined as follows: 
Definition 5: The degree of belief in the existence (absence) of an overlap relation between two messages $m_{i}$ and $m_{j}$ based on the criterion of their message activators is computed according to the function:

$$
\begin{aligned}
& \quad \mathrm{b}_{5}\left(\neg \mathrm{Ov}\left(\mathrm{m}_{\mathrm{i}}, \mathrm{m}_{\mathrm{j}}\right)\right)=\alpha_{5} \times \Sigma_{\mathrm{U} \subseteq\{1, \ldots, 4\}}(-1)^{|\mathrm{U}|+1}\left\{\Pi_{\mathrm{u} \in \mathrm{U}} \mathrm{b}_{\mathrm{u}}\left(\neg \mathrm{ov}\left(\mathrm{m}_{\mathrm{k}}, \mathrm{m}_{1}\right)\right)\right\} \text { if } \mathrm{m}_{\mathrm{k}} \neq \text { nil \& } \mathrm{m}_{1} \neq \text { nil } \\
& \quad \mathrm{b}_{5}\left(\neg \mathrm{Ov}\left(\mathrm{m}_{\mathrm{i}}, \mathrm{m}_{\mathrm{j}}\right)\right)=\alpha_{5} \times 1 \\
& \mathrm{~b}_{5}\left(\mathrm{ov}\left(\mathrm{m}_{\mathrm{i}}, \mathrm{m}_{\mathrm{j}}\right)\right)=0 \\
& \text { where } \\
& \text { - } \mathrm{m}_{\mathrm{k}} \text { and } \mathrm{m}_{1} \text { are the activators of the messages } \mathrm{m}_{\mathrm{i}} \text { and } \mathrm{m}_{\mathrm{j}} \text {, respectively } \\
& \text { - } \mathrm{b}_{1}, \ldots, \mathrm{b}_{4} \text { are the belief functions defined definitions } 1-4 \\
& \text { - } \alpha_{5} \text { is the expected ratio of messages with different activators which do not overlap }(0 \\
& \left.\leq \alpha_{5} \leq 1\right)
\end{aligned}
$$

According to Definition 5, the criteria used for computing beliefs in the absence of an overlap relation between the activators of two messages include the criteria of most generic overridden operation, operation stereotypes, message senders and message receivers but excludes the criteria of message activators and message activations (see Section 2.2.3). The reason for not using the criterion of message activators in the computation of $b_{5}$ beliefs is to avoid recursive computations in the transitive closure of the activators of the messages. Similarly, the reason for excluding the criterion of message activations when calculating beliefs in the existence and absence of an overlap relation between the activators of two messages $m_{i}$ and $m_{j}$ is that their inclusion would lead to a non terminating recursion in the computations, since it would require the computation of the belief in the absence of an overlap between $m_{i}$ and $m_{j}$ again (see definition of belief function $\mathrm{b}_{6}$ below).

\section{Examples}

Assuming that the parameters $\alpha_{1}, \alpha_{2}, \alpha_{3}, \alpha_{4}, \alpha_{5}, \alpha_{6}$ take the values $.4, .1, .2, .1, .2$, and .4 , respectively, the $b_{5}$ belief in the absence of an overlap relation between the messages 3:TextField("author_name") in $I_{1}$ and 2: TextField ("book_title") in $I_{2}$ is 0.02 . This is because there is weak evidence from the model against the existence of an overlap relation between the activators of these messages (these are the messages $2:$ new ( 01$)$ and 1 : new $(\mathrm{m})$, respectively). More specifically, in this case we have that:

$\Sigma_{\mathrm{U} \subseteq\{1, \ldots, 4\}}(-1)^{|\mathrm{U}|+1}\left\{\Pi_{\mathrm{u} \in \mathrm{U}} \mathrm{b}_{\mathrm{u}}(\neg \mathrm{ov}(2:\right.$ new $(\circ 1), 1$ : new $\left.(\mathrm{m})))\right\}=0.1$

\subsubsection{Equivalence of the implementations of operations}

\section{The criterion of message activations}

The final criterion for computing a partial belief in the existence/absence of an overlap relation between two messages $m_{i}$ and $m_{j}$ is whether the messages that $m_{i}$ and $m_{j}$ dispatch messages which overlap themselves or, in terms of UML, they have the same activations. This criterion is used since the dispatch of non-overlapping messages by two messages $m_{i}$ and $m_{j}$ implies that the operations which are invoked by $m_{i}$ and $m_{j}$ have different implementations. The belief function associated with this criterion is defined as follows: 
Definition 6: The degree of belief in the existence (absence) of an overlap relation between two messages $\mathrm{m}_{\mathrm{i}}$ and $\mathrm{m}_{\mathrm{j}}$ based on the criterion of message activations is computed according to the function:

where

$$
\mathrm{b}_{6}\left(\neg \mathrm{ov}\left(\mathrm{m}_{\mathrm{i}}, \mathrm{m}_{\mathrm{j}}\right)\right)=\alpha_{6} \times \mathrm{d}_{6}\left(\mathrm{~m}_{\mathrm{i}}, \mathrm{m}_{\mathrm{j}}\right) \quad \text { and } \quad \mathrm{b}_{6}\left(\mathrm{ov}\left(\mathrm{m}_{\mathrm{i}}, \mathrm{m}_{\mathrm{j}}\right)\right)=0
$$

- $\mathrm{d}_{6}\left(\mathrm{~m}_{\mathrm{i}}, \mathrm{m}_{\mathrm{j}}\right)=\left(\min _{\mathrm{X} \in \operatorname{Morphisms}(\mathrm{i}, \mathrm{j})}\left(\sum_{(\mathrm{mu}, \mathrm{mv}) \in \mathrm{X}} \mathrm{b}_{\mathrm{o}}\left(\neg \mathrm{Ov}\left(\mathrm{m}_{\mathrm{u}}, \mathrm{m}_{\mathrm{v}}\right)\right)+\max \left(\left|\mathrm{A}_{\mathrm{i}}\right|-\left|\mathrm{A}_{\mathrm{j}}\right|\right.\right.\right.$,

$$
\left.\left.\left|\mathrm{A}_{\mathrm{j}}\right|-\left|\mathrm{A}_{\mathrm{i}}\right|\right)\right) / \max \left(\left|\mathrm{A}_{\mathrm{i}}\right|,\left|\mathrm{A}_{\mathrm{j}}\right|\right) \quad \text { if } \mathrm{A}_{\mathrm{i}} \neq \varnothing \text { and } \mathrm{A}_{\mathrm{j}} \neq \varnothing
$$

$\mathrm{d}_{6}\left(\mathrm{~m}_{\mathrm{i}}, \mathrm{m}_{\mathrm{j}}\right)=1$

if $A_{i}=\varnothing$ or $A_{j}=\varnothing$

- $\mathrm{A}_{\mathrm{i}}$ and $\mathrm{A}_{\mathrm{j}}$ are the sets of messages which are dispatched by $\mathrm{m}_{\mathrm{i}}$ and $\mathrm{m}_{\mathrm{j}}$, respectively.

- Morphisms $(i, j)$ is the set of all the total morphisms from the messages in $\mathrm{A}_{\mathrm{i}}$ to the messages in $A_{j}$ if $\left|A_{i}\right| \leq\left|A_{j}\right|$ or onto morphisms from the messages in $A_{i}$ to the messages in $A_{j}$ if $\left|A_{j}\right|<\left|A_{i}\right|$.

- $\mathrm{b}_{0}\left(\neg \mathrm{Ov}\left(\mathrm{m}_{\mathrm{u}}, \mathrm{m}_{\mathrm{v}}\right)\right)$ is computed as defined by formula (I).

- $\alpha_{6}$ is the expected ratio of messages with different activations which do not overlap $\left(0 \leq \alpha_{6} \leq 1\right)$

According to Definition 6, the computation of $b_{6}$ beliefs for two messages $m_{i}$ and $m_{j}$ leads recursively to the identification of the most likely overlaps between all the messages which are directly or transitively dispatched by them (this is because $d_{6}$ is defined in terms of $b_{0}$ which, according to formula (I), is defined in terms of $b_{6}$ ). This recursive computation is terminated whenever the messages under comparison in the transitive closures of the activations of $m_{i}$ and $m_{j}$ dispatch no further messages. In cases where any of the messages under comparison dispatches no messages, $\mathrm{d}_{6}$ returns a belief equal to one. This belief reflects the hypothesis that in the absence of any evidence about the additional operations that two operations $\mathrm{o}_{1}$ and $\mathrm{o}_{2}$ invoke $\mathrm{o}_{1}$ and $\mathrm{o}_{2}$ can be assumed to have different implementations.

\section{Examples}

The $b_{6}$ beliefs in the absence of an overlap relation between the messages 1 : new $(\mathrm{m})$ and 2 :new $(01)$ and between the messages 7 :actionPerformed (ActionEvent) and 10 : actionPerformed (ActionEvent) in the interaction diagrams of Figure 1 are .88 and .61 , respectively. These beliefs are computed assuming that the parameters $\alpha_{1}, \alpha_{2}, \alpha_{3}, \alpha_{4}, \alpha_{5}, \alpha_{6}$ take the values $.4, .1, .2, .1, .2$, and .4, respectively.

The above $b_{6}$ beliefs reflect the facts that

(a) The activations of $1:$ new $(\mathrm{m})$ and 2 : new (o1) have only one pair of overlapping messages, that is the pair formed by the messages 2 :TextField("booktitle") and 3:TextField("author-name"), and four messages without overlapping counterparts, namely the messages 3 :addActionListener $(\mathrm{m})$, 4:Button("search"), 5:addActionListener (m), and 6 : setVisible (True) in the activation of 1 : new $(m)$.

(b) The activations of 7:actionPerformed (ActionEvent) and 10:actionPerformed(ActionEvent) have two pairs of overlapping messages (these are pairs formed by the messages 8:getText () and 
11:getText(), and the messages 10:executequery(SQLSt) and 12 : executequery (SQLStatement)), and one message without an overlapping counterpart, namely the message 9 : formulateQuery () .

\subsection{Example of detecting an overlap morphism}

The algorithm specified in Sections 2.1 and 2.2 detects the following overlapping messages in the interaction diagrams of Figure 1:

(i) message 2 : new (o1) in $I_{1}$ and message 1 : new $(m)$ in $I_{2}$

(ii) message 3:TextField ("author_name") in $\mathrm{I}_{1}$ and message 2 : TextField ("book-title") in $I_{2}$

(iii) message 4 : Button ("search") in $\mathrm{I}_{1}$ and message $4:$ Button ("search") in $\mathrm{I}_{2}$

(iv) message 10:actionPerformed (ActionEvent) in $\mathrm{I}_{1}$ and message 7 : actionPerformed (ActionEvent) in $\mathrm{I}_{2}$

(v) message 11 : get Text ( ) in $I_{1}$ and message 8 : get Text ( ) in $I_{2}$

(vi) message 12 : executeQuery (SQLStatement) in $I_{1}$ and message 10 : executeQuery (SQLSt) in $I_{2}$

\begin{tabular}{|l|c|c|c|c|c|c|c|}
\hline Messages & \multicolumn{7}{|c|}{ Beliefs } \\
\hline & $b_{1}$ & $b_{2}$ & $b_{3}$ & $b_{4}$ & $b_{5}$ & $b_{6}$ & $b_{o}$ \\
\hline$(2,1)$ & 0 & 0.214 & 0 & 0 & 1 & 0.928 & 0.507 \\
\hline$(3,2)$ & 0 & 1 & 0 & 0 & 0.52 & 1 & 0.516 \\
\hline$(4,4)$ & 0 & 1 & 0 & 0 & 0.52 & 1 & 0.516 \\
\hline$(10,7)$ & 0 & 0.612 & 0.214 & 0 & 0.479 & 0.616 & 0.388 \\
\hline$(11,8)$ & 0 & 0.214 & 0 & 0 & 0.1 & 1 & 0.424 \\
\hline$(12,10)$ & 0 & 0.214 & 0 & 0 & 0.1 & 1 & 0.424 \\
\hline
\end{tabular}

Table 1: Beliefs against the overlaps detected between the messages of $I_{1}$ and $I_{2}$

The beliefs in the absence of overlaps between the above messages are shown in Table 1 and were computed after setting the parameters $\alpha_{1}, \alpha_{2}, \alpha_{3}, \alpha_{4}, \alpha_{5}, \alpha_{6}$ and $b_{t}$ to 0.4 , $0.1,0.2,0.1,0.2,0.4$, and 0.65 , respectively. The rows of the table designate the above pairs of messages by using the numbers that indicate the order of their dispatch in each diagram. For example, the overlapping messages 11:get Text () and 8 : get Text ( ) are signified in Table 1 as the pair $(11,8)$.

The values selected for the parameters $\alpha_{1}-\alpha_{6}$ in this example were empirically determined after considering models that incorporated generalisation graphs and object interactions specified at varying degrees of completeness and elaboration. Reconciliation+ assumes that designers should decide which are the appropriate values for the parameters $\alpha_{1}-\alpha_{6}$ and $b_{t}$. These decisions should be based on an assessment of how accurate is as an indicator of overlaps each of the criteria that underpin the belief functions $b_{1}-b_{6}$. This assessment can be formed based on the form and level of elaboration of different parts of the involved design model(s). If a model uses no 
stereotypes, for instance, $\alpha_{4}$ should be set to 0 . Similarly, for models that do not incorporate elaborate class generalisation graphs $\alpha_{2}$ should be set to a low value.

\subsection{Properties of the belief functions}

As proved in [21], the functions $b_{1}-b_{6}$ are all distance metrics and satisfy the axioms of Dempster-Shafer basic probability assignments [19]. The functional form of $b_{0}$ is derived from the combination of the belief functions $b_{1}, \ldots, b_{6}$ using the rule of the orthogonal sum of the Dempster-Shafer theory, and measures the belief that is jointly committed to $\neg \mathrm{OV}\left(\mathrm{m}_{\mathrm{i}}, \mathrm{m}_{\mathrm{j}}\right)$ by $\mathrm{b}_{1}-\mathrm{b}_{6}$. $\mathrm{b}_{0}$ is also a distance metric (see [21] for a proof). These characteristics of $b_{0}$ guarantee the following intuitive properties for its outputs:

- for any three messages $m_{i}, m_{j}$ and $m_{k}$ we have, due to the triangularity of distance metrics, that: $\mathrm{b}_{0}\left(\neg \mathrm{ov}\left(\mathrm{m}_{\mathrm{i}}, \mathrm{m}_{\mathrm{k}}\right)\right) \leq \mathrm{b}_{0}\left(\neg \mathrm{ov}\left(\mathrm{m}_{\mathrm{i}}, \mathrm{m}_{\mathrm{j}}\right)\right)+\mathrm{b}_{0}\left(\neg \mathrm{ov}\left(\mathrm{m}_{\mathrm{j}}, \mathrm{m}_{\mathrm{k}}\right)\right)$

- for any two messages $m_{i}$ and $m_{j}$ we have, due to the symmetry of distance metrics, that: $\mathrm{b}_{0}\left(\neg \mathrm{ov}\left(\mathrm{m}_{\mathrm{i}}, \mathrm{m}_{\mathrm{j}}\right)\right)=\mathrm{b}_{0}\left(\neg \mathrm{ov}\left(\mathrm{m}_{\mathrm{j}}, \mathrm{m}_{\mathrm{i}}\right)\right)$

- for any two messages $m_{i}$ and $m_{j}$ we have, due to axiomatic foundation of Dempster-Shafer basic probability assignments [19], that: $b_{0}\left(\neg 0 v\left(m_{i}, m_{j}\right) \wedge\right.$ $\left.\operatorname{ov}\left(\mathrm{m}_{\mathrm{j}}, \mathrm{m}_{\mathrm{i}}\right)\right)=0$

\section{The Reconciliation+ process: specification and enactment}

As we discussed in Section 1, Reconciliation+ guides software designers through the activity of reconciling their models by enacting a built-in process model. This model specifies consistency rules that may be checked against overlapping messages and alternative ways of handling violations of these rules. In this section, we introduce the scheme that is used by the method to specify this process model, and the mechanism that is used to enact it.

\subsection{A UML profile for specifying reconciliation processes}

The process of Reconciliation+ is specified as a graph of contexts following a decision-oriented approach to software process modelling [11]. A context represents a decision that may be taken in a given situation. This situation is specified as a condition over the state of the software models which are being manipulated by the process (i.e., the interaction diagrams which are being reconciled in the case of Reconciliation+). Contexts are distinguished into:

(1) executable contexts - these are contexts which represent decisions to take actions that change the state of the software model;

(2) plan contexts - these are contexts which represent decisions that can be realised by a set of sub-decisions which must be made in a specific order; and 
(3) choice contexts - these are contexts which represent decisions that may be realised by two or more other alternative decisions.

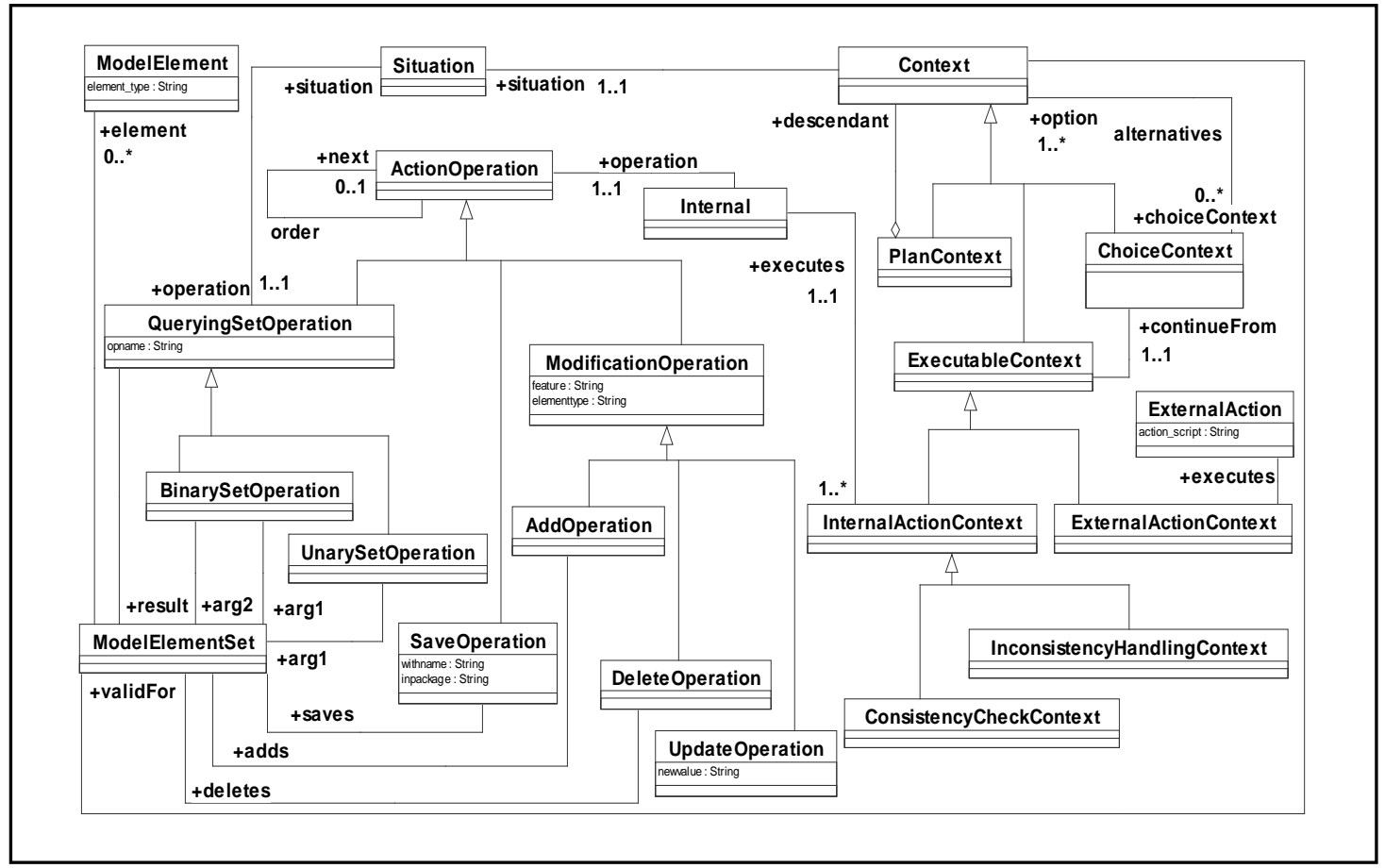

Figure 3: A UML profile for specifying Reconciliation+ processes

The process models that can be formulated using this process modelling approach are essentially AND/OR graphs of decisions which are guarded by specific conditions. Figure 3 presents a UML profile that we have defined for specifying model reconciliation processes in UML using the contextual process modelling approach described above ${ }^{5}$.

Profiles in UML are user-defined extensions of the meta-model of the language which are introduced to support the construction of models for specific purposes and domains. Typically, a UML profile consists of: (i) a set of stereotypes that define additional kinds of modelling elements which are required to construct models for the particular domain, (ii) tag definitions that introduce additional meta-attributes for the model elements that may be classified under the stereotypes of the profile, and (iii) constraints which are associated with the stereotypes of the profile and apply to all the elements classified under them [9].

The Reconciliation+ process specification profile that we have developed is represented by the UML class diagram shown in Figure 3. This diagram has been constructed according to the following representation conventions:

\footnotetext{
5 The introduction of a UML profile for the specification of the reconciliation processes of our method was motivated by the need to have both software models and the processes that reconcile them expressed in a single representational framework as this would facilitate the implementation of tool support for the method (see Section 6).
} 
(i) Classes represent the stereotypes of the profile. The class ChoiceContext, for example, designates the stereotype that represents choice contexts in the process modelling approach outlined above.

(ii) A named association end in Figure 3 designates a tag defined for the stereotype that is represented by the class that is attached to the opposite end of the relevant association. For example, situation is a tag defined for the stereotype Context. The type of this tag is the stereotype Situation and its multiplicity is $1 . .1$.

(iii) An attribute in Figure 3 designates a tag defined for the stereotype that is represented by the class incorporating it. Attributes represent tags whose type is a data type. For example, feature is a tag defined for the stereotype Modificationoperation whose type is String.

(iv) A generalisation relation in Figure 3 designates a generalisation relation between the stereotypes represented by the classes that it connects. For example, ChoiceContext is a special kind of Context.

As shown in Figure 3, our profile, includes stereotypes that represent the basic constructs for specifying reconciliation processes, including contexts, situations and actions.

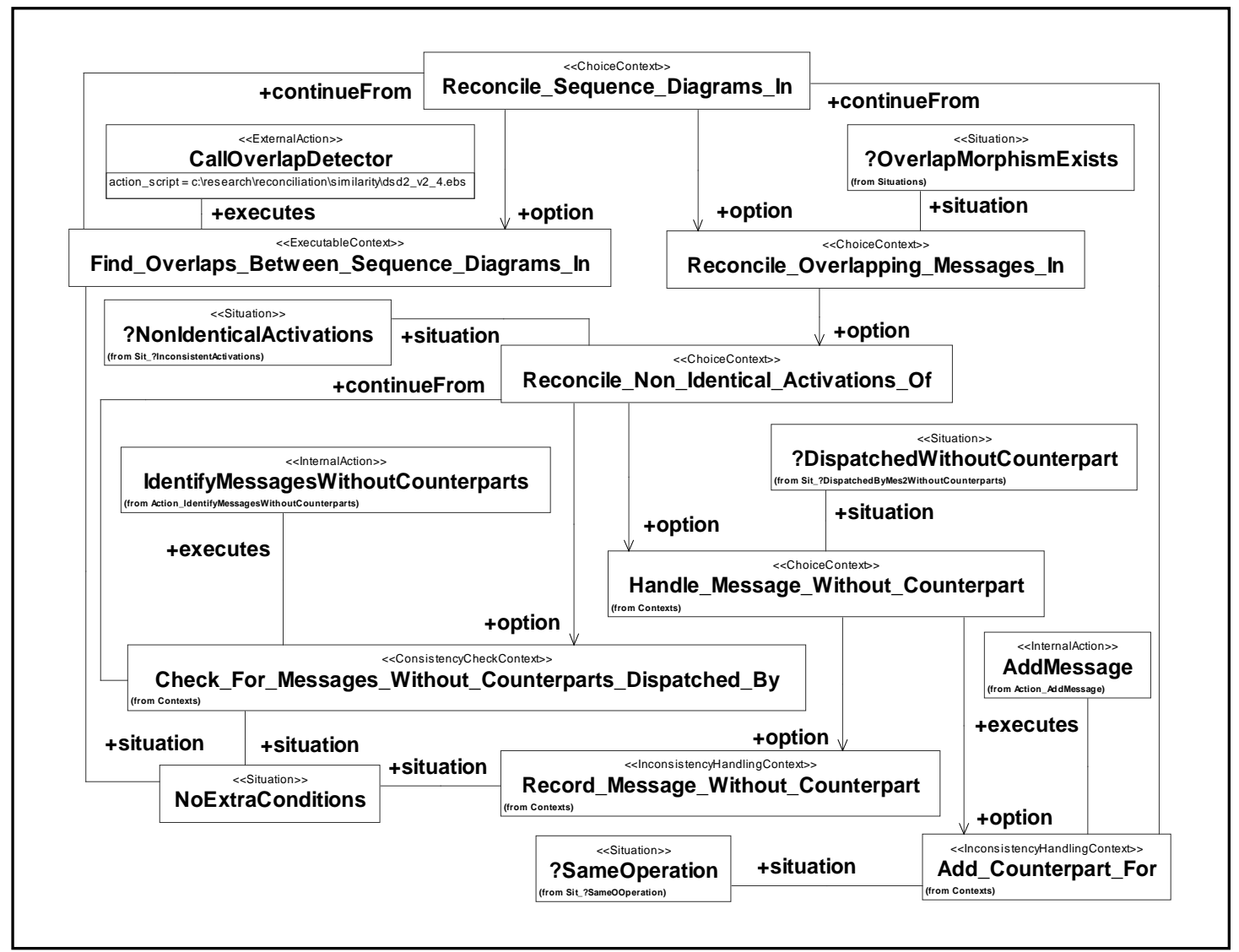

Figure 4: Part of the Reconciliation+ process model 
A part of the Reconciliation+ process model that is specified according to the above profile is shown in Figure 4. This part includes, for example, the choice context Reconcile_Overlapping_Messages_In which represents the decision to start the process of reconciling overlapping messages. The situation of this context, ?OverlapMorphismExists, checks whether an overlap morphism between the interaction diagrams to be reconciled has been identified. Thus, the context Reconcile_Overlapping_Messages_In can be selected only if the overlaps between two interaction models have been identified.

\subsection{Specification of situations}

In Reconciliation+, a situation is specified as a query defined by an ordered sequence of querying set operations. A querying set operation is either a Unarysetoperation or a BinarySetoperation.

Unary set operations are used to retrieve elements of a UML model which are associated with a given element $e$ of it via any of the different kinds of associations or attributes defined for the type of $e$. A unary set operation is associated with two sets: the $\arg 1$ set and the result set. The former set includes all the elements of a UML model that the operation should be applied to. The latter set is used to store the union of the elements which are retrieved for each of the elements of arg1 following the application of the operation. An example of a unary set operation is the operation imwnc-02 in Figure 6. This operation is stereotyped as a GetActivation operation, that is a unary set operation which retrieves the messages which are directly dispatched by the messages which belong to its arg1 set. Additional examples of unary set operations are given in Section 4.

Binary set operations are associated with three sets: the $\arg 1, \arg 2$ and result set. There are three different types of such operations in our process specification profile for computing the union, intersection and set-difference of given sets. Similarly to unary operations, the result of a binary set operation is saved in its result set. An example of a binary set operation is the operation imwnc-o7 in Figure 6. This operation is stereotyped as a SetDifference operation and, therefore, it computes the set difference of the sets of messages imwnc-o6-s 1 and imwnc-o4-s1 (i.e., imwnc-o6-s1 - imwnc-o4-s1) and inserts the elements of this set difference in imwnc-07-s1.

The operations that define the query of a situation $s$ are ordered through the association order ${ }^{6}$ (see Figure 3) and may take as an argument any of the sets which are generated by operations preceding them in $s$. A situation is satisfied if the result set of the last of its operations is not empty. Examples of specifications of situations are given in Section 5.

${ }^{6}$ The association end next of this association denotes the next operation in a local operation sequence. 


\subsection{Specification of actions}

In our process specification profile, each executable context must have an action that can be either an external or internal action (see the action stereotypes ExternalAction and InternalAction in Figure 3).

External actions are used to specify the invocation of external tools during the enactment of a Reconciliation+ process. An example of an external action is the action CalloverlapDetector in the process model of Figure 4. This action is associated with the context Find_Overlaps_Between_Sequence_Diagrams_In and is executed when this context is selected. The execution of this action invokes the overlap detection tool of Reconciliation+ (a pointer to the executable file of this tool is specified as the value of the attribute action_script of the action as shown in Figure 4).

Internal actions are used to specify consistency rules and ways of handling inconsistencies. These actions are specified as sequences of action operations. An action operation may be a querying, model modification, or save operation. Querying operations are the same as those used in the specification of situations. Modification operations are operations which are used to modify the state of the software models being manipulated by the process. Save operations are used to store the results of querying operations in the trace of the enactment of a process model so as to make them available in subsequent stages of this enactment. Action operations are ordered in internal actions and executed similarly to sequences of querying operations in situations. Examples of specifications of internal actions and the different types of operations that may be used in them are given in Sections 4 and 5.

\subsection{Process enactment}

The Reconciliation+ process model is enacted by an engine which functions as a model interpreter [13]. The algorithm underpinning the operation of this engine is specified in Figure 5. According to this algorithm, the enactment of a process model starts from the root context of the process model (a process model must have a single root context that should be a choice context). The situation of the root context of a process model (and any other context that is encountered as the enactment engine traverses it) is evaluated by executing the set querying operations that define it. If the set which results from the execution of the last of these operations (called situation set) is not empty, the situation of the context is considered to have been satisfied. In this case, the enactment engine generates different possible decisions from the context, one for each of the elements in the situation set.

More specifically, a decision is defined as a pair:

where

$$
\text { <contexti, situation_set_element }{ }_{j}>
$$

- context $i$ is the context whose situation is satisfied, and 
- situation_set_element $t_{j}$ is an element of the situation set of context ${ }_{i}$.

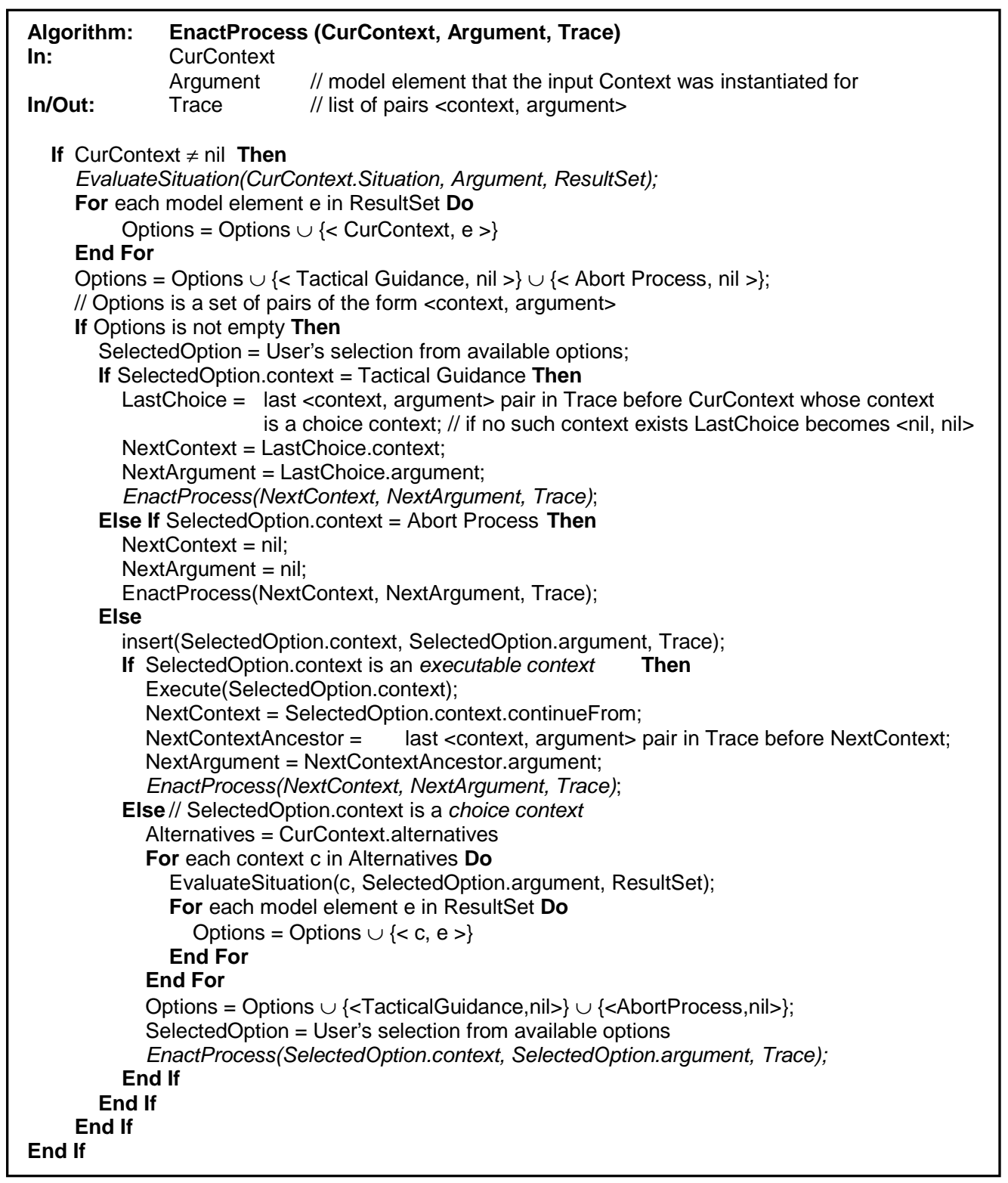

Figure 5: Process enactment algorithm

The situation ?NonIdenticalActivations in the process model of Figure 4, for example, retrieves all the pairs of overlapping messages of two interaction diagrams that have non identical activations. In the case of the interaction diagrams $I_{1}$ and $I_{2}$, these pairs of messages are:

(2: new (o1), 1 : new $(m))$

(10:actionPerformed (ActionEvent), $7:$ actionPerformed (ActionEvent))

Thus, the possible decisions that may be generated from the context Reconcile_Non_Identical_Activations_of of this situation when reconciling $I_{1}$ and $I_{2}$ are: 
(1) <Reconcile_Non_Identical_Activations_of, (2:new (o1),

$1: \operatorname{new}(\mathrm{m}))>$

(2) <Reconcile_Non_Identical_Activations_of,

(10:actionPerformed (ActionEvent),

7 :actionPerformed (ActionEvent)) >

A designer may select one of the different possible decisions which are generated from a context, ask for tactical guidance or terminate the process. If a decision <context , situation_set_element $_{j}>$ is selected, it is recorded in the trace of the enacted process model and subsequently:

- If context $t_{i}$ is a choice context, the enactment engine: (1) retrieves the option contexts associated with it, (2) inserts the situation_set_element $t_{j}$ in the $\arg 1$ set of the initial querying set operation of each of these contexts, (3) evaluates the situation of each of these contexts, (4) generates the possible decisions for each of these contexts, and (5) prompts the designer to make a new selection.

- If context $t_{i}$ is an external action context, the enactment engine executes the file specified by the attribute action_script of it and continues the enactment of the process model from the context associated with context $i$ via the association end continueFrom (see Figure 3).

- If context $i$ is an internal action context, the enactment engine executes the sequence of the operations in its internal action and continues the enactment of the process model as in the case of external action contexts. ${ }^{7}$

In cases where the designer asks for tactical guidance, the enactment engine identifies the decision before the last decision recorded in the process trace and resumes execution from the context of it. The designer may also abort the execution of the process model at any point.

In the following, we describe how the process specification profile of our method can be used to specify consistency rules and actions to handle their violations.

\section{Detection of inconsistencies}

In Reconciliation+, a consistency rule is defined as an internal action of a consistency check context (i.e., a special kind of executable contexts as shown in Figure 3). This action is essentially a query which retrieves the model elements that violate the conditions required by the rule. Thus, the specification of consistency rules is procedural. The consistency check context which incorporates the internal action that defines a consistency rule represents the decision to check the rule. It also specifies the conditions under which this decision may be made. These conditions are specified by the situation of the context.

7 Plan contexts are not used in the current process model of Reconciliation+ and therefore the description of their enactment is beyond the scope of this paper. 
Furthermore, the internal actions of consistency check contexts are restricted not to include any modification operations. This restriction guarantees that the execution of a consistency rule will not modify the contents of the underlying models. Also, the last operation of such actions must be a save operation that records the model elements which violate the rules (see stereotype Saveoperation in Figure 3) in order to make them available to subsequent stages of the enactment of the reconciliation process.

Figure 6 shows the internal action IdentifyMessagesWithoutCounterparts that defines the consistency rule CR1. As discussed in Section 1, CR1 requires that if a message $m_{i}$ overlaps with a message $m_{j}$ then for each message x activated by $m_{i}$ there must be a message $\mathrm{y}$ activated by $m_{j}$ that overlaps with $\mathrm{x}$ and vice versa. The specification of this internal action assumes that message overlap relations are represented by overlap objects in the trace of the Reconcilication+ process which point to the overlapping messages and store the beliefs in their overlap (see the object

10 : actionPerformed (ActionEvent) $\leftrightarrow 7$ : actionPerformed (ActionEvent) in Figure 7).

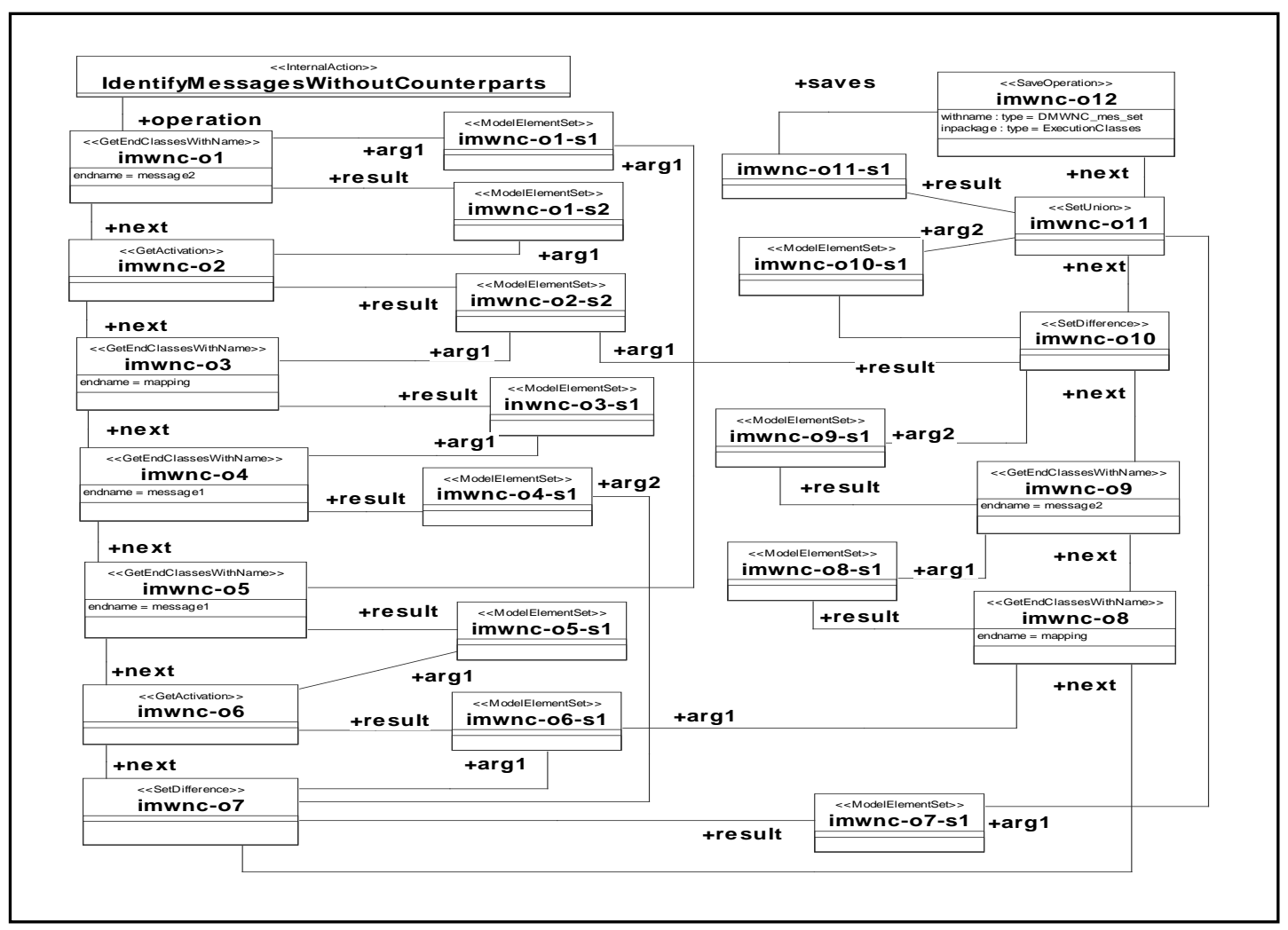

Figure 6: Specification of the internal action IdentifyMessagesWithoutCounterparts

To implement CR1, IdentifyMessagesWithoutCounterparts retrieves the messages in the activations of two overlapping messages which do not have overlapping counterparts through the execution of the following operations: 
(1) $i m w n c-o 1^{8}-$ this operation retrieves one of the messages that is pointed to by the selected overlap object (message2).

(2) imwnc-o2 - this is an operation which, by virtue of its stereotype (i.e., a GetActivation operation), finds the messages which are directly dispatched by a message (message 2 of the selected overlap object in this case).

(3) imwnc-o3 and imwnc-o4 - these operations find the messages that overlap with the messages dispatched by message 2 and inserts them in set imwnc-o4s1.

(4) imwnc-05 and imwnc-06 - these operations find the messages which are directly dispatched by the other message that is pointed to by the selected overlap object (i.e., message1) and insert them in set imwnc-o6-s1.

(5) imwnc-07 - this operation finds the messages which are dispatched by message 1 and have no counterparts in the set of messages dispatched by message 2 by computing the difference between set imwnc-o6-s1 and set imwnc-o4-s 1 .

(6) imwnc-08, imwnc-09, and imwnc-010 - similarly to steps 4-5 these operations identify the messages which are dispatched by message 2 and have no counterparts in the set of messages dispatched by message 1.

(7) imwnc-011 - this operation takes the union of the messages which are dispatched by message1 and message2 and have no overlapping counterparts.

(8) imwnc-012 - this operation saves the messages which are dispatched by message1 and message 2 and have no overlapping counterparts as elements of the set DMWNC_mes_set.

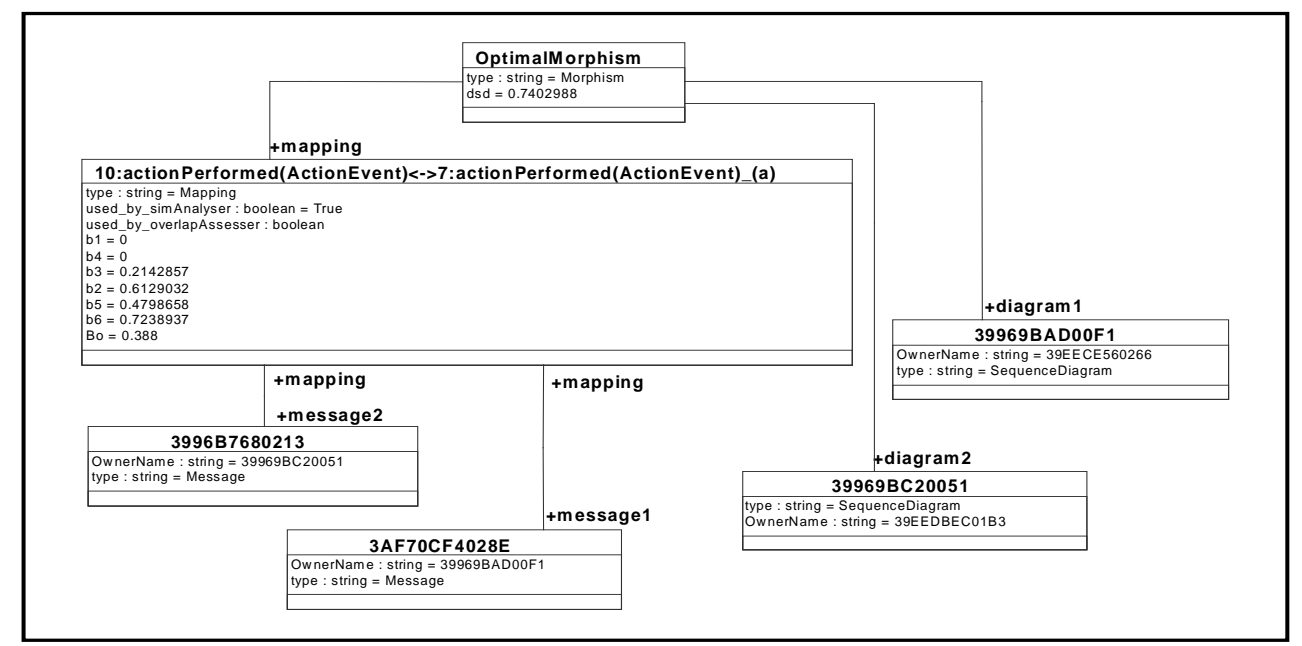

Figure 7: Overlap objects - objects that represent overlap relations

IdentifyMessagesWithoutCounterparts is specified as the internal action of the consistency check context

8 imwnc-o1 is an operation stereotyped as GetEndClassesWithName. Operations of this stereotype retrieve the objects which are related to the elements in their arg 1 set via the association end named by their attribute endname. 
Check_For_Messages_Without_Counterparts_Dispatched_By (see Figure 4). Thus, to check the consistency rule CR1 against a pair of overlapping messages, a designer has to decide to apply this context to this pair. Note, however, that Check_For_Messages_Without_Counterparts_Dispatched_By may be applied only in certain parts of the reconciliation process and if the situation associated with it is satisfied. More specifically, according to the process model of Figure 4 , this context can be applied to a pair of overlapping messages only after a designer has selected:

1) the executable context

Find_Overlaps_Between_Sequence_Diagrams_In to detect overlaps in the interaction diagrams to be reconciled;

2) the choice context Reconcile_Overlapping_Messages_In to start the reconciliation of the overlapping messages detected in these interaction diagrams; and

3) the choice context Reconcile_Non_Identical_Activations_of to start the reconciliation of the overlapping messages with the non-identical activations.

The selection and application of the consistency check context Check_For_Messages_Without_Counterparts_Dispatched_By to the overlap object of Figure 7 (that is the object 10: actionPerformed (ActionEvent) $\leftrightarrow 7$ : actionPerformed (ActionE vent)) leads to the execution of the internal action IdentifyMessagesWithoutCounterparts which retrieves and saves in the process trace the following set of messages without overlapping counterparts:

DMWNC_mes_set $=\{9$ : formulateQuery ()$\}$

\section{$5 \quad$ Handling inconsistencies}

The ways of handling inconsistencies in Reconciliation+ are specified as internal actions of a special kind of executable contexts, called inconsistency handling contexts (see stereotype InconsistencyHandlingContext in Figure 3).

A consistency rule is associated with one or more inconsistency handling contexts which specify alternative ways of handling its violations. These contexts are grouped as options of a choice context which, by virtue of the definition of its situation, becomes selectable only if there is a record of violations of the particular consistency rule in the trace of the reconciliation process. The alternative inconsistency handling contexts which are available as options of this context are associated with situations which define the particular conditions under which the alternative inconsistency handling options may be applied.

In the following, we discuss how the situations and the actions of inconsistency handling contexts can be specified using the process modelling profile of 
Reconciliation+. Our discussion is based on inconsistency handling contexts that the process model of Reconciliation+ incorporates to deal with inconsistencies which arise as violations of the rule CR1.

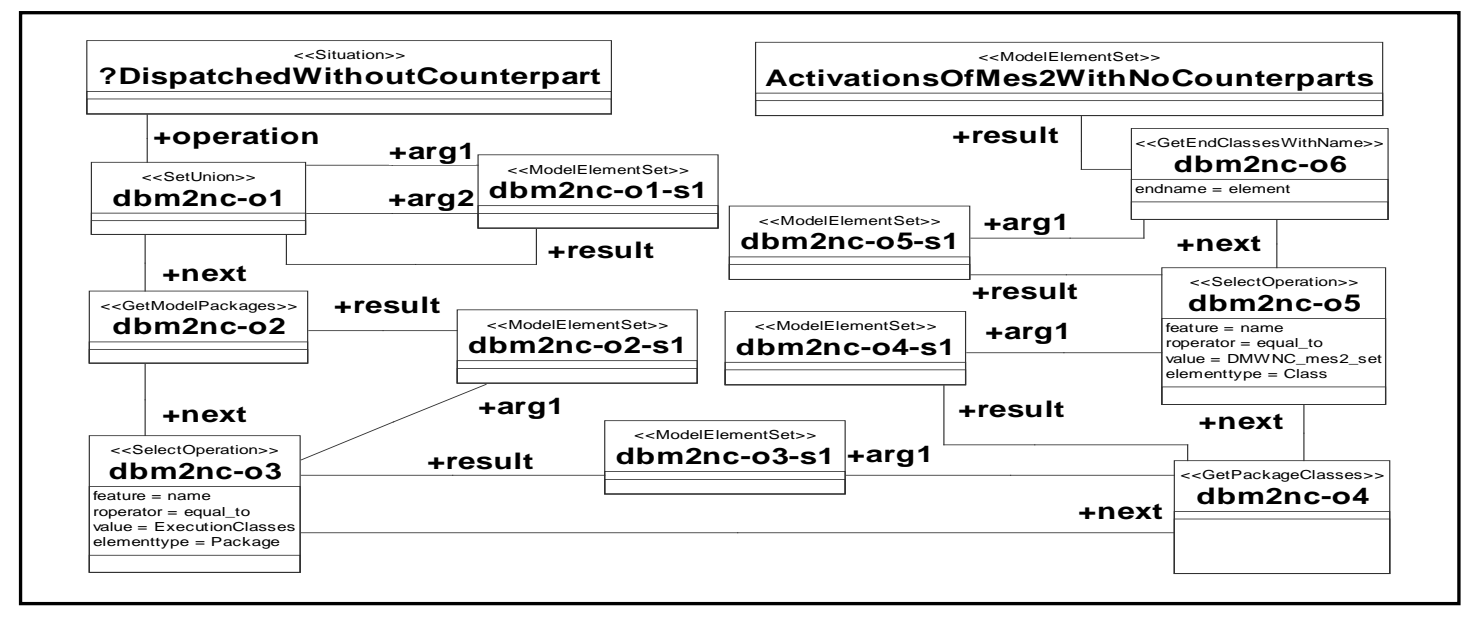

Figure 8: Specification of situation ?DispatchedWithoutCounterparts

A violation of $\mathrm{CR} 1$ can be handled in different ways. One possibility, for instance, is to delete the messages without counterparts from the relevant message activation. A second possibility is to add the missing messages in the relevant activation. A third possibility is to modify the software models so that overlapping messages that dispatch messages without counterparts will no longer be considered as overlapping messages. A fourth possibility is to record the inconsistency and postpone its resolution.

The process model shown in Figure 4 includes the contexts Add_Counterpart_For and Record_Message_Without_Counterpart, which correspond to the $2^{\text {nd }}$ and $4^{\text {th }}$ of the above options. As shown in Figure 4 , these contexts are grouped as options of the choice context Handle_Message_With_No_Counterpart. The latter context, due to the definition of its situation ?DispatchedWithoutCounterparts (see Figure 8), becomes available only if there are messages that violate CR1. This is because, according to Figure 8, ?DispatchedWithoutCounterparts is satisfied only if there is a non empty set called DMWNC_mes_set that has as elements the messages in the activations of two overlapping messages that violate $\mathrm{CR} 1^{9}$ (recall from Section 4 that DMWNC_mes_set is generated by the action of the context Check_For_Messages_Without_Counterparts_Dispatched_By).

Handle_Message_Without_Counterpart can be applied to any of the messages in DMWNC_mes_set. These alternative applications are generated as decisions by the process enactment engine as described in Section 3.4 and are proposed to the designer. In the case of the overlap relation between the messages 10 : actionPerformed (ActionPerformed)

and

9 The set DMWNC_mes_set is represented as an object that is associated with all its elements and exists in a special package called ExecutionClasses of the repository of the Reconciliation+ toolkit. 
7 :actionPerformed (ActionEvent) of the interaction diagrams $I_{1}$ and $I_{2}$ the only decision generated from Handle_ Message_Without_Counterpart is: <Handle_Message_Without_Counterpart, 9: formulatequery()>

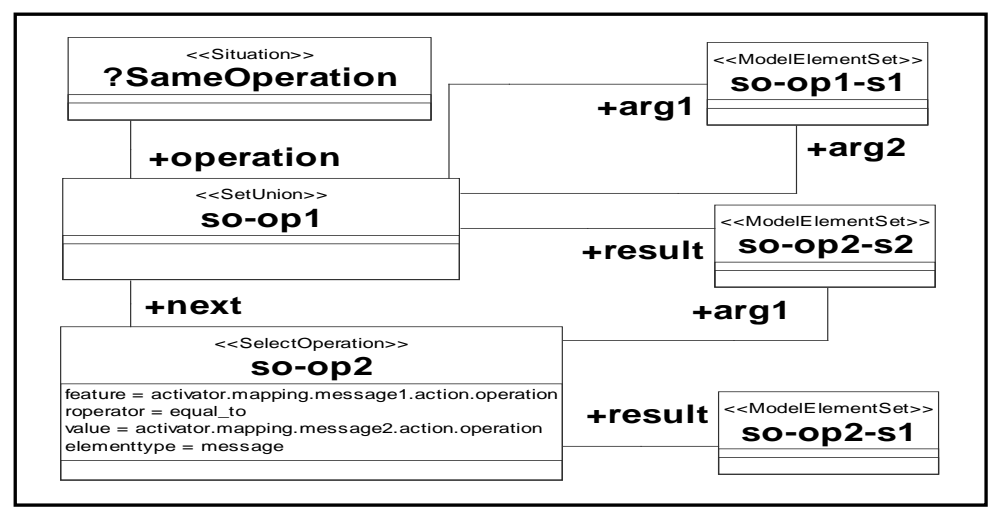

Figure 9: Specification of situation ?SameOperation

If this decision is selected, the process enactment engine checks the situations of the two alternative contexts for handling this particular kind of inconsistencies, namely Record_Message_Without_Counterpart and Add_Counterpart_For to establish if they can be applied in the case of 9 : formulateQuery ( ) .

If applicable, Record_Message_Without_Counterpart can be activated to make a persistent record of the inconsistency (i.e., a record saved after the end of the reconciliation process). This context can be selected under any circumstances as its situation contains no conditions in addition to those set by its ancestor context in the process model.

Add_Counterpart_For can be selected to create a copy of a message that does not have an overlapping counterpart and add it to the activation of the message that overlaps with its activator. Note, however, that this way of resolving the inconsistency makes sense only if the overlapping messages that gave rise to it indeed invoke the same operation in the object model. Thus, the situation of Add_Counterpart_For (i.e., the situation ?SameOperation shown in Figure 9) is specified so as to check whether this is the case. More specifically, the unary set operation so-op2 in ?SameOperation retrieves the activator $m l$ of the message that caused the violation of CR1 (i.e., 9: formulateQuery () in our example) and the message $m 2$ that $m 1$ overlaps with and checks if $m 1$ and $m 2$ have been declared in the models to invoke the same operation (the operations invoked by $m 1$ and $m 2$ are identified through the evaluation of the path expressions activator.mapping.message1.action.operation and activator.mapping.message 2 action.operation ${ }^{10}$ ). If that is the case,

\footnotetext{
${ }^{10}$ The evaluation of the sub-paths activator.mapping.message $1(2)$ in these path expressions locate the overlapping messages in the activations of which the message without the counterpart was encountered. These sub-paths assume the representation of overlap morphisms and relations by the tool that we have built to support Reconciliation+ (shown in Figure 7). The remaining sub-paths
} 
?SameOperation is satisfied and, therefore, Add_Counterpart_For can be selected for the message 9 : formulateQuery ().

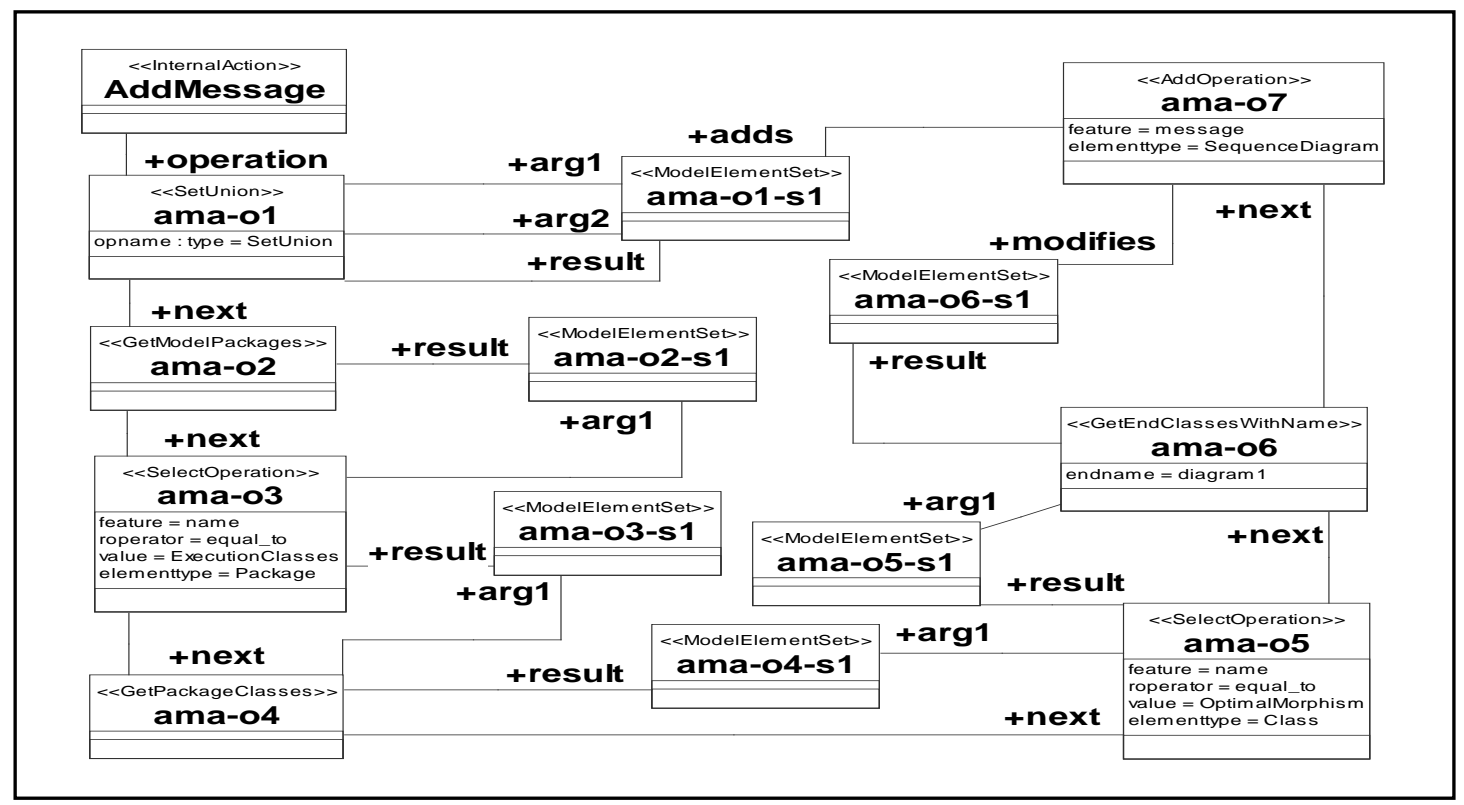

Figure 10: Specification of action AddMessage

The creation of a copy of this message is the result of executing the internal action AddMessage of Add_Counterpart_For. The specification of this action is shown in Figure 10. According to this specification, to execute AddMessage, the process enactment engine first locates the interaction diagram of the overlapping message of the activator of the message that Add_Counterpart_For was selected for (i.e., the diagram $I_{1}$ in our example) by executing the operations ama-०2 to ama-06, and then adds to the set of the messages of this diagram a copy of this message by executing the operation ama-07.

\section{Tool support}

Reconciliation+ is supported by a toolkit which incorporates: (a) a tool that detects overlaps between object interaction models, and (b) an engine which enacts the process model of the method to drive the activity of reconciling interaction diagrams. This toolkit has been implemented as an add-on utility for Rational Rose (a CASE tool supporting UML) using the API of this tool [21].

The architecture of the Reconciliation+ toolkit is shown in Figure 11. As shown in this figure, the toolkit stores the models to be reconciled as collections of UML class models and sequence diagrams in a model repository that is accessible through the API of Rose. The overlap morphisms which are detected by the overlap detection tool,

(*.action.operation) assume the standard representation for UML models that is established by the UML meta-model [9]. 
the process model of the method, and the trace of the enactment of this model are also represented and stored as UML object models in the same repository.

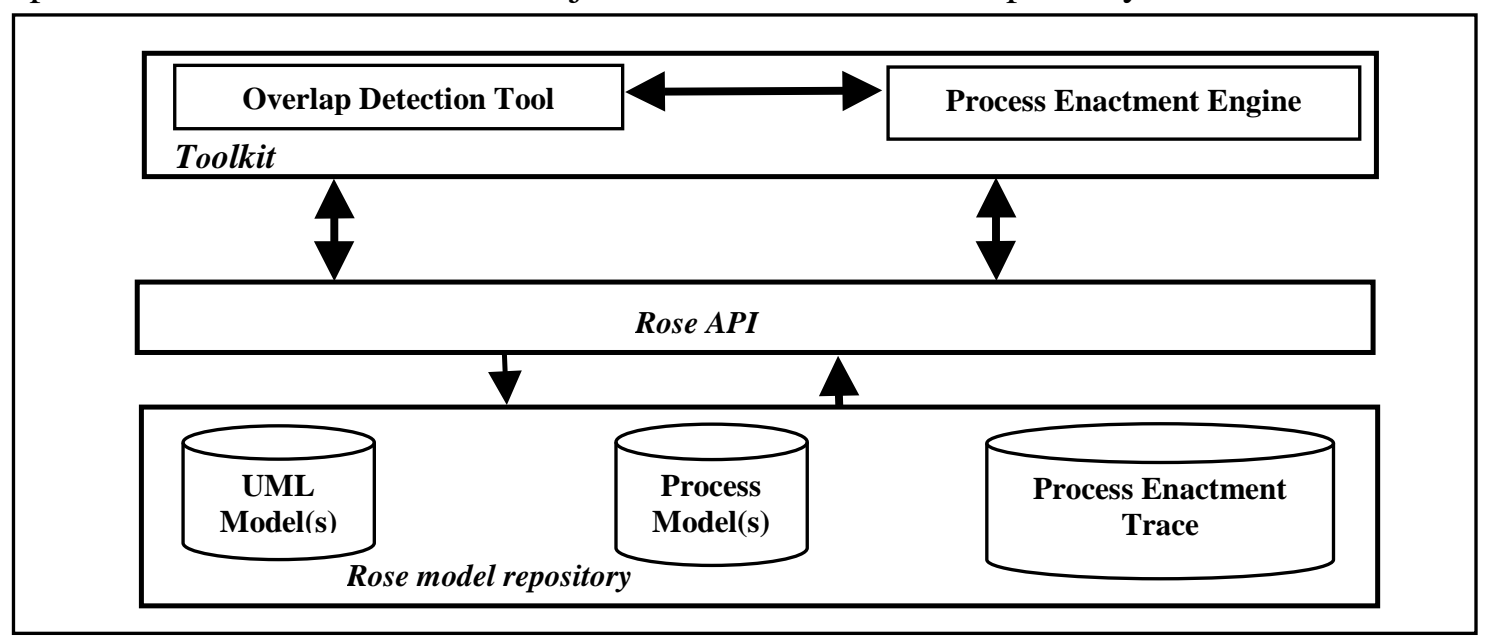

Figure 11: Architecture of the Reconciliation+ toolkit

Figure 12 shows a snapshot of the overlap detection tool following its invocation to identify overlaps between the interaction diagrams of Figure 1.

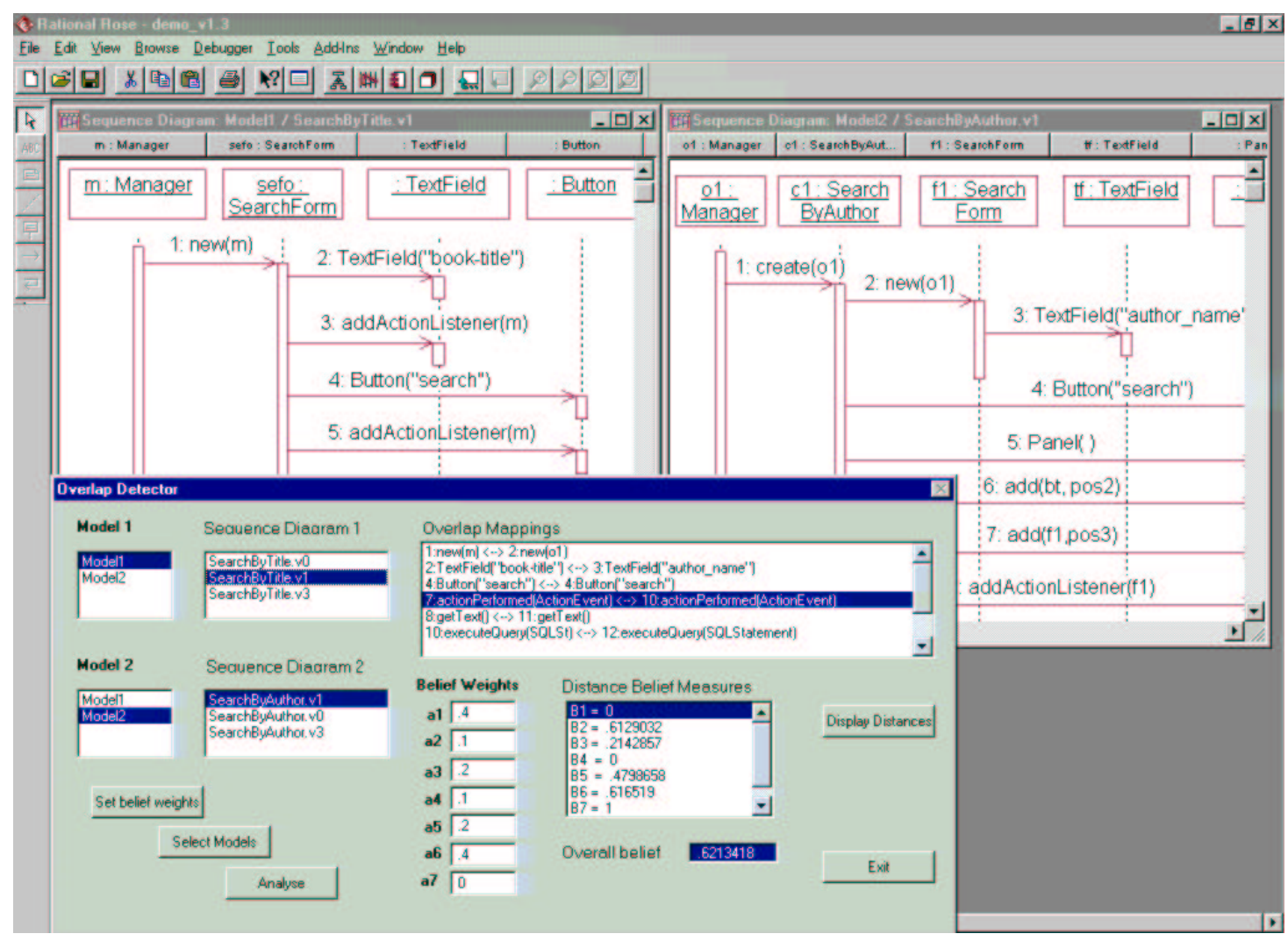

Figure 12: Overlap Detector - detection of overlap morphism between $I_{1}$ and $I_{2}$

Figure 13 shows a snapshot of the process enactment engine of the toolkit. This snapshot shows a point in the enactment of the Reconciliation + process model where the consistency

check

context 
Check_For_Messages_Without_Counterparts_Dispatched_By may be selected to check for violations of the rule CR1 by messages in the activations of the overlapping pair of messages: 10 :actionPerformed (ActionEvent), and 7 :actionPerformed (ActionEvent) of the diagrams of Figure 1. As shown in Figure 13, the process enactment engine gives a designer the options of: (a) applying any of the contexts which become available at the current point in the enactment of the process model (see list Next Decision), and (b) asking for tactical guidance or equivalently go back to the previous decision point in the enactment of the process model. Note also that the enactment engine keeps a record of the decisions that have been made up to the current point in the enactment of a process (process trace) and presents them to the designer (see the list Decisions made so far).

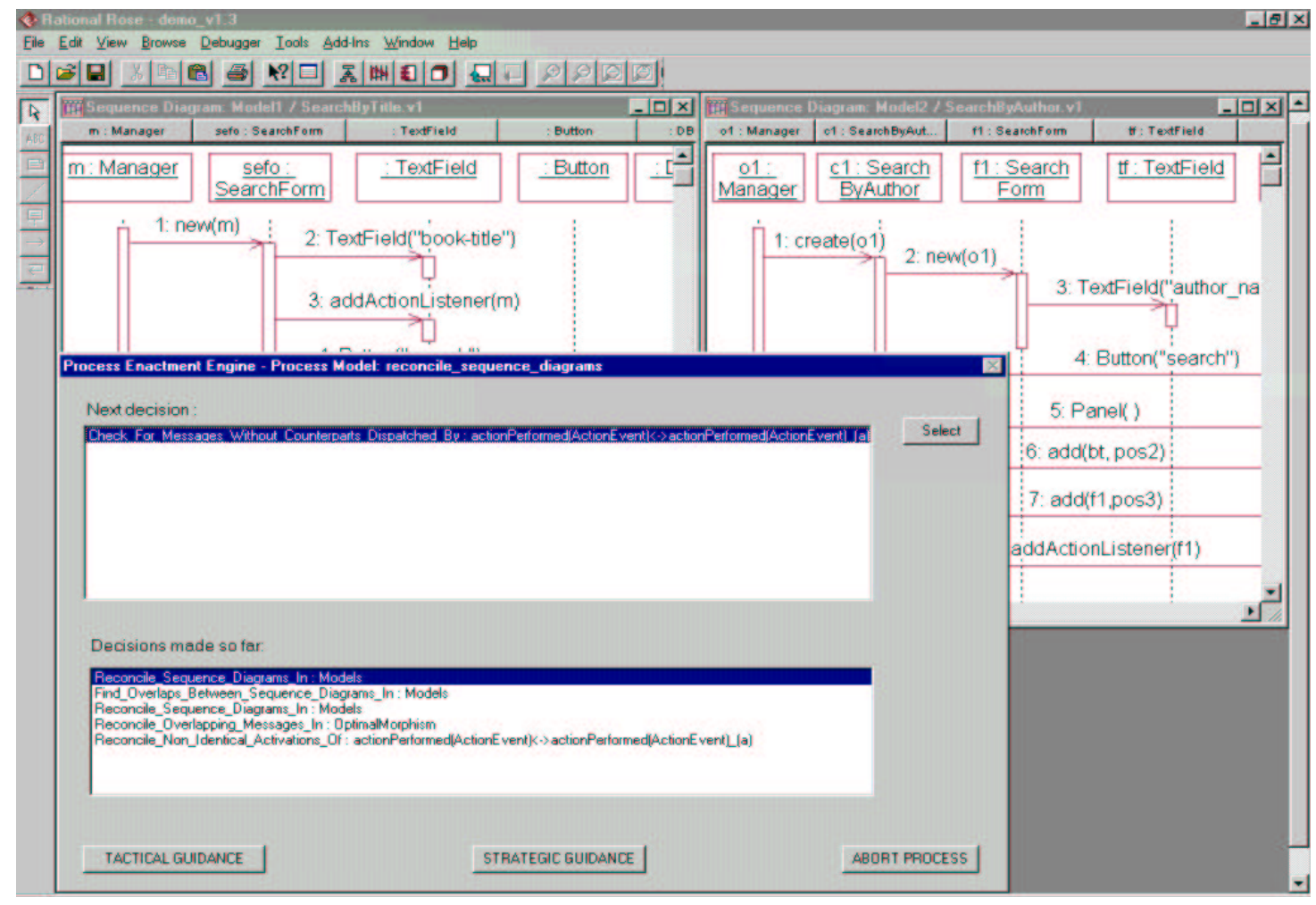

Figure 13: Process Enactment Engine

\section{$7 \quad$ Case study}

To evaluate Reconciliation+ we have carried out a case study. The objectives of this study were to:

(i) measure the performance of the overlap detection algorithm that is deployed by the method in terms of recall (i.e., the ability to detect overlap relations that humans would identify), and precision (i.e., the ability to detect correct overlap relations);

(ii) assess the sensitivity of the overlap detection algorithm against the granularity of the models it is applied to; and 
(iii) investigate the diversity of the ways that may be used to handle inconsistencies, and assess whether the process model of the method could support the specification of inconsistency handling contexts to implement these ways.

In this case study, we used 5 UML models built by MSc students at City University. These models had been constructed to specify a system supporting banking transactions through the use of ATM machines.

\subsection{Recall and precision of overlap detection algorithm}

To evaluate the recall and precision of the overlap detection algorithm of Reconciliation+, we performed 50 comparisons of pairs of UML interaction diagrams drawn from the models of our case study.

Following a manual identification of overlap relations between these interaction diagrams, we detected overlap relations between them using the algorithm specified in Section 2, and subsequently measured the recall and the precision of the algorithm according to the following formulas:

where

$$
\text { Precision }=\frac{\left|\mathrm{AO}^{\mathrm{ij}} \cap \mathrm{MO}^{\mathrm{ij}}\right|}{\left|A \mathrm{O}^{\mathrm{ij}}\right|} \text { and Recall }=\frac{\left|\mathrm{AO}^{\mathrm{ij}} \cap \mathrm{MO}^{\mathrm{ij}}\right|}{\left|\mathrm{MO}^{\mathrm{ij}}\right|}
$$

- $\mathrm{AO}^{\mathrm{ij}}$ is the set of the overlap relations that were detected by the overlap detection algorithm between the messages of two interaction diagrams $i$ and $j$; and

- $\mathrm{MO}^{\mathrm{ij}}$ is the set of the manually identified overlap relations between the messages of two interaction diagrams $i$ and $j$.

\begin{tabular}{|c|c|c|c|c|c|c|}
\hline Model & $\mid$ MO $\cup$ AO & \#ID-Pairs & \multicolumn{2}{|c|}{ Recall } & \multicolumn{2}{c|}{ Precision } \\
\hline & & & Mean & St. Dev. & Mean & St. Dev. \\
\hline 1 & 77 & 10 & 1.00 & 0 & 1.00 & 0 \\
\hline 2 & 60 & 11 & 0.86 & 0.20 & 0.99 & 0.03 \\
\hline 3 & 74 & 6 & 0.92 & 0.20 & 0.90 & 0.2 \\
\hline 4 & 231 & 15 & 0.87 & 0.08 & 0.98 & 0.05 \\
\hline 5 & 87 & 8 & 0.91 & 0.06 & 1.00 & 0 \\
\hline All & 529 & 50 & 0.91 & 0.13 & 0.98 & 0.08 \\
\hline
\end{tabular}

Table 2: Recall and precision of overlap detection algorithm

Table 2 shows the average and standard deviation of the recall and precision measures that we obtained for pairs of diagrams drawn from each of the five object models used in our experiments, and from all of the models (these results were obtained for the values of parameters $\alpha_{1}-\alpha_{6}$ and $b_{t}$ used in Section 2.3). It also shows the number of the pairs of interaction diagrams that were compared in each model (i.e., \#ID-Pairs) and the total number of the overlap relations detected by the overlap algorithm and the overlap relations that were manually identified between them in each case (i.e., |MO $\cup$ $\mathrm{AO} \mid)$. The object models that we used in our experiments and the overlap relations identified manually by the experts and the overlap detection algorithm are available from: http://www.soi.city.ac.uk/ gespan/imoosd_case_studies.html 
As shown in Table 2, the overlap detection algorithm had very high precision ( 0.98 on average across all models) and relatively high recall (0.91 on average across models). Also there was a low variation in these measures across different pairs of interaction diagrams and models (the standard deviation of the recall and precision measures across all models were 0.13 and 0.07 , respectively). Although preliminary, the above results indicate that the overlap detection algorithm has a very low probability of producing false overlaps and is capable of detecting a high proportion of the overlaps indicated by human designers.

\subsection{Effect of model granularity on overlap detection}

To explore the effect of model granularity on our overlap detection algorithm, we also carried out a correlation analysis of the obtained recall and precision measures against the following measures of model granularity:

(i) the number of classes in a model (\#CL) - this measure was expected to affect the beliefs $b_{3}$ and $b_{4}$,

(ii) the average number of superclasses of a class in a model (\#Isa*) - this measure was expected to affect directly the beliefs $b_{2}$ and $b_{3}$ and implicitly $b_{5}$,

(iii) the average degree of operation overriding in a model (i.e., the ratio of classes which inherit an operation but override it - \#OO) - this measure was expected to affect directly the beliefs $b_{1}$ and implicitly $b_{5}$, and

(iv) the average number of dispatched messages in message activations (\#AC) - this measure was expected to affect the beliefs $b_{6}$.

\begin{tabular}{|c|c|c|c|c|}
\hline Model & \#Cl & \#Isa* & \#OO & \#AC \\
\hline 1 & 697 & 0.014 & 1 & 3.685 \\
\hline 2 & 200 & 0.420 & 0.305 & 1.288 \\
\hline 3 & 55 & 0.309 & 0.584 & 2.722 \\
\hline 4 & 702 & 0.017 & 0.772 & 1.030 \\
\hline 5 & 696 & 0.012 & 0.696 & 1.911 \\
\hline Correlation Coefficients \\
\hline Recall & 0.29 & -0.28 & 0.76 & 0.96 \\
\hline Precision & 0.74 & -0.74 & 0.24 & -0.16 \\
\hline
\end{tabular}

Table 3: Model granularity measures and correlation with recall and precision

Table 3 presents the above granularity measures for the five models of our case study and their correlation with the recall and precision measures obtained for these models. As shown in the table, recall had strong positive correlations with \#OO and \#AC. This was expected as the weights of the $\mathrm{d}_{1}$ and $\mathrm{d}_{6}$ distances, which were directly affected by \#OO and \#AC, in establishing beliefs in overlaps were relatively higher than the weights of the other four distances of our algorithm (see values of $\mathrm{a}_{1}$ and $\mathrm{a}_{6}$ in Section 2.3). The observed negative correlation of recall with the average number of superclasses (\#Isa*) is likely to have been the result of the small number of superclasses that the senders and receivers of messaged had in the considered models. It should also be noted that only the positive correlation of \#AC with recall (i.e., 0.96) was statistically significant (at $\alpha=0.10$ ). 
In the case of precision, positive correlations were detected only for \#CL and \#OO and none of the obtained correlations was statistically significant at $\alpha=0.10$. This may be attributed to the fact that precision was very high across all models and therefore the differences in model granularity did not have any significant effect on it.

The results of the above correlation analysis indicate that the overlap detection algorithm is not over-sensitive to the degree of completeness and elaboration of design models. This was expected due to the use of six different criteria that focus on different parts of software design models for detecting overlaps. Clearly, however, our results are only preliminary and need to be confirmed by additional experiments.

\subsection{Diversity of inconsistency handling options}

The third objective of our case study was to investigate the diversity of the ways that may be used to handle inconsistencies, and to assess whether the process model of the method could support the specification of inconsistency handling contexts to implement these ways. In this part of the study, we selected 4 consistency rules to check against the overlapping messages that were detected by the overlap detection algorithm in the first part of the study. The selected rules were:

- CR1 - this was the rule specified in Sections 1 and 4;

- CR2 - this was a rule that required the operations invoked by two overlapping messages to have the same name;

- CR3 - this was a rule that required the operations invoked by two overlapping messages to be defined in the same class; and

- CR4 - this was a rule that required the operations invoked by two overlapping messages to have the same number and types of parameters.

The above rules were selected since they capture the main forms of possible discrepancies in the specifications of operations which are invoked by overlapping messages.

\begin{tabular}{|l|c|c|c|c|}
\hline & CR1 & CR2 & CR3 & CR4 \\
\hline Violations & 40 & 3 & 2 & 23 \\
\hline No need to be resolved & 10 & 0 & 1 & 9 \\
\hline Need to be resolved & 29 & 3 & 1 & 14 \\
\hline Alternative resolutions & 6 & 2 & 1 & 4 \\
\hline
\end{tabular}

Table 4: Inconsistencies and alternative ways of handling them

Table 4 shows the number of the detected violations of each of these rules, the number of the cases where we found that it was necessary to resolve the detected inconsistency, and the number of the different alternative ways that we advocated for resolving the inconsistencies of the same rule. As it may be seen from the figures of this table, some diversity was indeed observed in the ways of handling violations of the same rule. However, due to the size of our experiments these results cannot be generalised. Nevertheless, the case study has given rise to some interesting observations that we discuss in the following. 
More specifically,

- In some cases, following the violation of a consistency rule, it was realised that the overlap relation detected by the method and checked against the rule was wrong. In such cases, it was not necessary to take any inconsistency resolution action. The way that was adopted to handle such inconsistencies was to record them along with an annotation that the overlap relation that gave rise to them were wrong.

- There were cases, where the violation of a consistency rule should be tolerated as the relevant rule should not be satisfied by particular pairs of overlapping messages. This was, for instance, the case with some of the violations of rule CR1 in which two overlapping messages were dispatching messages with the same signature but different activations which had not themselves been detected as overlapping messages. In these cases, the non overlapping messages were invoking polymorphic operations (i.e., operations defined in different classes with the same signature and different implementations as, for example, the operations SearchForm.actionPerformed (e: ActionEvent) and DatabaseActionListened.actionPerformed (e: ActionEvent) in Figure 2) and therefore the resolution of the inconsistency was not necessary. To deal with such cases, it is, in principle, possible either to amend the conditions of the relevant rule so as to ignore non overlapping messages that invoke polymorphic operations, or to add inconsistency handling contexts for the rule that ignore its violations by such messages. Although both these strategies can be accommodated by the method, Reconciliation+ does not incorporate at its current stage of development criteria for helping designers to decide which of the two options is more appropriate in specific circumstances.

- The selection of the best inconsistency handling strategy in a given situation may depend on the satisfiability (or unsatisfiability) of more than one rules. The violation of both rule CR1 and CR2, for instance, in some cases led to the realisation that the overlap relation that gave rise to the inconsistencies was wrong. In these cases, the relevant inconsistencies were ignored. In other cases, however, where CR3 was violated but CR1 was satisfied the inconsistencies were resolved by changing the name of one of the overlapping messages. In Reconciliation+, cases like these could be handled by specifying the situations of the inconsistency handling contexts for a specific rule so as to check if the pair of the overlapping messages that violated the rule has also violated another rule.

\section{Related work}

A considerable body of research has been concerned with the problem of detecting inconsistencies in software models and documentation. This work has generated techniques for detecting inconsistencies in structured and text-based [1][3][4][12] object-oriented [2][6][15][18] state-based [7][8], and formal software models [5][17].

Some of the proposed techniques focus on object-oriented models. Glinz [6], for example, has developed a technique that checks behavioural software models expressed as statecharts for deadlocks, reachability and mutual exclusiveness of states. 
Cheung et al [2] have developed a technique that checks whether the sequence of the execution of operations that is implied by a UML statechart diagram is compliant with the sequence of the executions of operations implied by a UML sequence diagram. Zisman et al [18] have developed a consistency link generator which checks whether UML software models satisfy specific consistency rules. These rules are expressed in XML and the consistency checking is performed using a tool developed using an XML development platform. A critical survey of all the above techniques may be found in [16].

\section{Conclusions and further work}

In this paper, we have presented Reconciliation+, a method that guides designers in reconciling object interaction diagrams specified as part of software design models. The method detects overlaps between messages in interaction diagrams, checks consistency rules that overlapping messages must satisfy, and provides ways of handling violations of these rules. The rules and the ways of handling inconsistencies are specified as parts of a process model that is enacted by the method to drive the reconciliation activity. Reconciliation+ can be applied at the design phase of software development following the specification of, at least, partial object-oriented software design models defining the basic class structure and interactions of a system. The method can be used in conjunction with development approaches which require the development of design models prior to implementation and approaches that advocate an incremental development of such models and/or software systems.

We have evaluated Reconciliation+ in a case study the objectives of which were to measure the recall and precision of the overlap detection algorithm deployed by the method, investigate its sensitivity to variations of model granularity, and investigate the diversity of the strategies that may be needed in handling inconsistencies. This case study has shown positive preliminary results regarding the recall and precision of the overlap detection algorithm of the method, and has demonstrated some diversity in the nature of inconsistency handling strategies which can be accommodated by the method. It has also shown that the overlap detection algorithm is not prohibitively sensitive to the degree of elaboration and completeness of the models it is applied to. Thus, Reconciliation+ can be applied to models specified at varying levels of completeness.

Further experimentation is, however, required to confirm these findings. Furthermore, it is necessary to evaluate the method against some usability criteria including the difficulty in extending its process model with new consistency rules and ways of handling their violations, as well as with intermediate decisions (choice contexts) to guide software designers in selecting amongst alternative inconsistency handling options. Currently, we are evaluating the method along these lines.

\section{Acknowledgements}

The work presented in this paper has been partially funded by the EPSRC grant no. GR/M57422. 


\section{References}

[1] Boehm B, In H (1996), Identifying Quality Requirements Conflicts, IEEE Software, 25-35.

[2] Cheung K, Chow K, Cheung T (1998), Consistency Analysis on Lifecycle Model and Interaction Model, Proc. of the $7^{\text {th }}$ Int. Conference on Object-Oriented Information Systems, 427-441.

[3] Easterbrook S (1991), Handling Conflict between Domain Descriptions with Computer-Supported Negotiation, Knowledge Acquisition, 3: 255-289.

[4] Emmerich W, Finkelstein F, Montangero C, Antonelli S, Armitage S (1999), Managing Standards Compliance, IEEE Transactions on Software Engineering, 25(6): 836-851.

[5] Finkelstein A., Gabbay D, Hunter, A, Kramer, J, and Nuseibeh, B (1994), Inconsistency Handling In MultiPerspective Specifications, IEEE Transactions on Software Engineering, 20(8): 569-578.

[6] Glinz M (1995), An Integrated Formal Model of Scenarios Based on Statecharts, In Proc. of the $5^{\text {th }}$ European Software Engineering Conference, LNCS 989, Springer-Verlag, 254-271.

[7] Heimdahl M.P.E, Leveson N (1996), Completeness and Consistency in Hierarchical State-Based Requirements, IEEE Transactions in Software Engineering, 22(6): 363-377.

[8] Heitmeyer C, Jeffords R, Kiskis D (1996), Automated Consistency Checking Requirements Specifications, ACM Transactions on Software Engineering and Methodology, 5(3): 231-261.

[9] OMG, Unified Modeling Language Specification (Action Semantics) - V. 1.4. Available from: http://www.omg.org/technology/documents/modeling_spec_catalog.htm .

[10] Papadimitriou C, Steiglitz K (1982), Combinatorial Optimisation: Algorithms and Complexity, Prentice-Hall Inc.

[11] Pohl K (1996), Process-Centred Requirements Engineering, Advanced Software Development Series, J. Kramer (ed), Research Studies Press Ltd., ISBN 0-86380-193-5, London.

[12] Robinson, W. and Fickas S (1994), Supporting Multi-Perspective Requirements Engineering, In Proc. of the IEEE Conference on Requirements Engineering, IEEE Computer Society Press, 206-215.

[13] Si-Said S, Rolland C, Grosz G (1996), MENTOR: A Computer Aided Requirements Engineering Environment, Proc. of the $8^{\text {th }}$ International Conference on Advanced Information Systems Engineering, 22-43.

[14] Spanoudakis G, Constantopoulos P (1996), Elaborating Analogies from Conceptual Models, International Journal of Intelligent Systems, 11(11): 17-974.

[15] Spanoudakis G, and Finkelstein A (1997), Reconciling requirements: a method for managing interference, inconsistency and conflict, Annals of Software Engineering, Special Issue on Software Requirements Engineering, 3: 459-475.

[16] Spanoudakis G, Zisman A. (2001), Inconsistency Management in Software Engineering: Survey and Open Research Issues, Handbook of Software Engineering and Knowledge Engineering, (ed) Chang S. K, World Scientific Publishing Co, 329-380.

[17] Lamsweerde A, Darimont R, Letier E (1998), Managing Conflicts in Goal-Driven Requirements Engineering, IEEE Transactions on Software Engineering, 24(11): 908-926.

[18] Zisman A, Emmerich W, Finkelstein A (2000), Using XML to Specify Consistency Rules for Distributed Documents, Proc. of $10^{\text {th }}$ Int. Workshop on Software Specification and Design.

[19] Shafer G (1976), A Mathematical Theory of Evidence, Princeton University Press.

[20] http://java.sun.com/j2se/1.3/docs/guide/awt/

[21] Spanoudakis G (2000), An Algorithm for Detecting Overlaps between Models of Object Interactions, Technical Report Series, TR-2000/03, ISSN 1364-4009, Department of Computing, City University.

[22] http://www.rational.com/products/rose/index.jsp. 